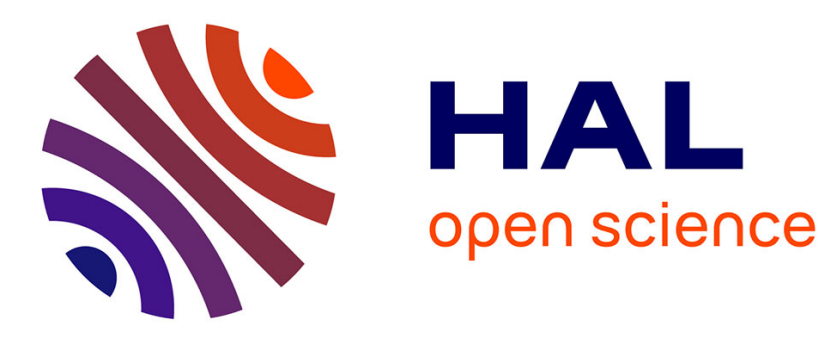

\title{
Mixing and chaos in open flows
}

\author{
Alessandro de Moura, Ulrike Feudel, Emmanuelle Gouillart
}

\section{To cite this version:}

Alessandro de Moura, Ulrike Feudel, Emmanuelle Gouillart. Mixing and chaos in open flows. 2011. hal-00614299

\section{HAL Id: hal-00614299 \\ https://hal.science/hal-00614299}

Preprint submitted on 10 Aug 2011

HAL is a multi-disciplinary open access archive for the deposit and dissemination of scientific research documents, whether they are published or not. The documents may come from teaching and research institutions in France or abroad, or from public or private research centers.
L'archive ouverte pluridisciplinaire HAL, est destinée au dépôt et à la diffusion de documents scientifiques de niveau recherche, publiés ou non, émanant des établissements d'enseignement et de recherche français ou étrangers, des laboratoires publics ou privés. 


\title{
Mixing and chaos in open flows
}

\author{
Alessandro Moura ${ }^{\mathrm{a}, *}$, Ulrike Feudel $^{\mathrm{b}}$, Emmanuelle Gouillart $^{\mathrm{c}}$ \\ ${ }^{a}$ Institute of Complex Systems and Mathematical Biology, King's College, University of \\ Aberdeen, Aberdeen AB24 3UE, UK \\ ${ }^{b}$ Institut für Chemie und Biologie des Meeres, Carl von Ossietzky Universität, PF 2503, \\ D-26111 Oldenburg, Germany \\ ${ }^{c}$ Surface du Verre et Interfaces, UMR 125 CNRS/Saint-Gobain, 93303 Aubervilliers, \\ France
}

\begin{abstract}
We review the theory and experimental knowledge of mixing in open flows displaying chaotic advection, from a point of view of dynamical systems theory. We show that the chaotic saddle and its stable and unstable manifolds constitute the skeleton around which the dynamics are organised, and that their fractal properties govern advection and mixing in open flows. The effects of KAM islands on the mixing is examined, as well as the interplay between molecular diffusion and chaotic advection. We discuss what the appropriate definition of mixing is in practical situations, and present experiments motivated by industrial applications to back these discussions. We also discuss applications of these concepts to plankton dynamics in the oceans.
\end{abstract}

Keywords: Chaotic advection, Open flows, Mixing

\section{Introduction}

When one thinks of mixing, the image most likely to come to mind is that of a liquid being stirred in a closed container. This is indeed a very important scenario, which has been the focus of most studies on the dynamics of mixing since Aref's pioneering work (Aref, 1984). That landmark work and those which followed have established that stirring can give rise to chaotic advection, which results in the continuous stretching and folding

\footnotetext{
*Corresponding author

Email address: a.moura@abdn.ac.uk (Alessandro Moura)
} 
of any given portion of the fluid. This chaotic dynamics causes any blob of fluid to be quickly deformed into a filamentary shape with very thin filaments spreading all over the container (see Fig. 1). As the stirring continues, the filaments become denser and denser in space as their total length increases, until molecular diffusion mixes them homogeneously into the background. This homogeneous mixing is the direct result of the fact that the container is closed: the filaments are continuously stretched, and having nowhere else to go, they end up filling the entire space in the container.

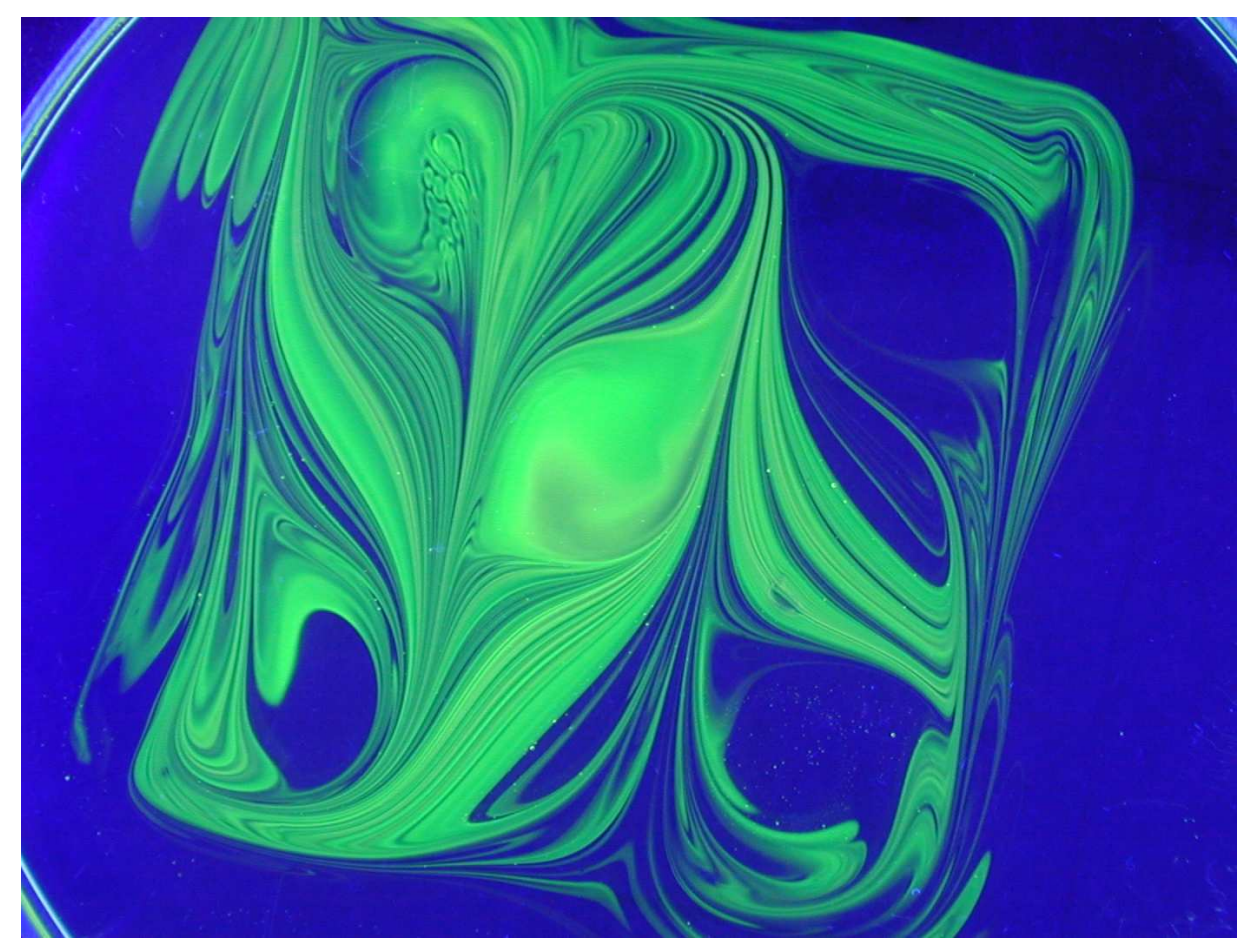

Figure 1: Shape of a dye droplet after stirring on the surface of a thin layer of glycerol in a Petri dish. Experiment carried out by I.M. Jánosi, K.G. Szabó, T. Tél, and M. Wells at the von Kármán Laboratory of Eötvös University, Budapest. From (Tél et al., 2005).

The study of mixing in open flows - flows with constant inflow and outflow, where fluid is not bound to a region of finite volume - has been less thoroughly pursued. But open flows are extremely important in many areas of science and engineering, and problems involving mixing in open flows are 
crucial for many natural and man-made systems. Chaotic advection is also present in open flows, such as in the flow of streams around obstacles, and in situations where flow is stirred in the open, without walls restricting the motion of the fluid. In open flows, chaos takes a different form compared to the case of closed flows (Tél et al., 2005), because typical fluid particles escape any given region of space in a finite time. There is, however, a set of unstable "trapped" orbits which are bounded within a finite region and which never escape. These non-escaping orbits constitute an invariant set of the dynamical system associated with advection - the chaotic saddle. Even though the orbits in the chaotic saddle have zero measure in space - that is, the probability that an initial condition chosen randomly belongs to one of the trapped orbits in the chaotic saddle is zero - , they are very important for understanding the dynamics of open systems, because they determine the asymptotic $(t \rightarrow \infty)$ advection dynamics. The chaotic saddle has a fractal geometry, and this gives rise to complex behaviour of fluid parcels in their neighbourhood. Fluid particles near the chaotic saddle undergo successive episodes of stretching and folding, just as in the closed container case. But the difference is that the flow is escaping as well, and as a result the filaments produced by stretching are not space-filling. The fractal structures generated by the chaotic dynamics are limited by the outflow, and the situation of homogeneous mixing found in the closed case is never reached in open flows; instead a characteristic pattern of filaments with intricate structure reflecting their underlying fractal geometry is formed, as illustrated in Fig. 2, which shows the simulation of passive tracers being advected in a human blood vessel (Schelin et al., 2009, 2010). An experimental example of a dye mixed into an open fluid, a problem of great industrial interest, which is described in Section 6.

It is clear that in an open system the flow is not mixed in the sense of the closed case, since some of the fluid escapes through the outflow unmixed. But the portions of fluid whose trajectories bring them close to the chaotic saddle are stretched and folded many times before they escape, and in those regions some mixing does take place. In other words, in open flows there is partial mixing, due to the transient nature of the dynamics of open flows.

The importance of open flows for so many areas makes it important to have a solid understanding of the dynamics of mixing in those systems. In this work, we review the most important results in this area, and present the major relevant concepts in a unified framework, and we also discuss some of the most relevant applications of these concepts in physics and engineering. 


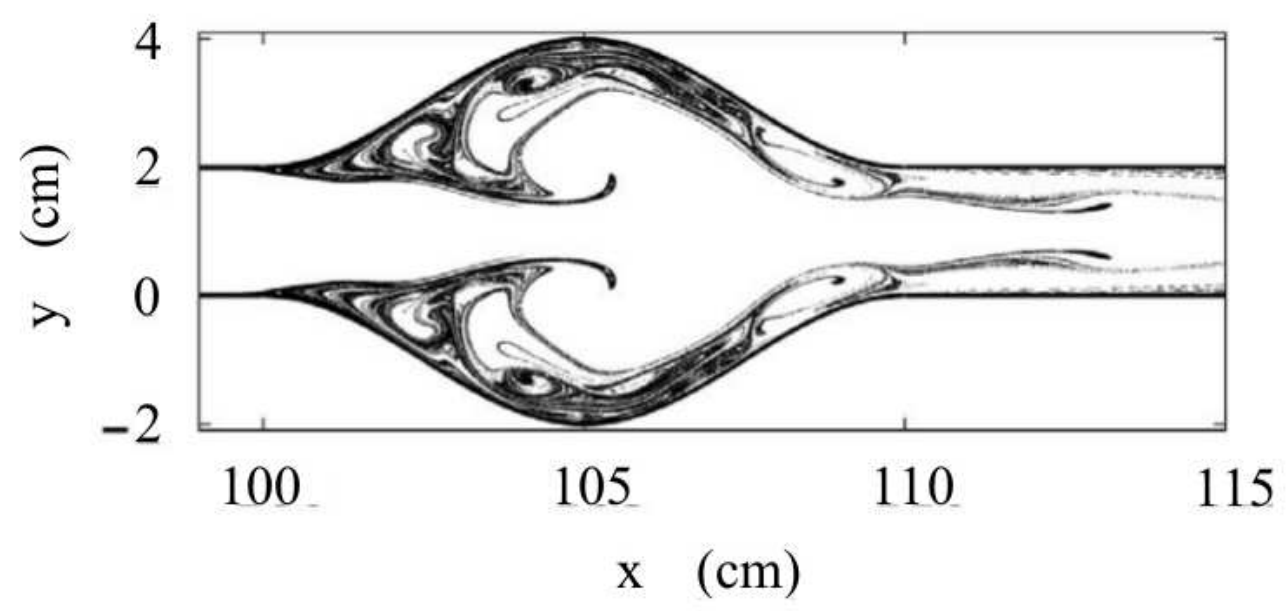

Figure 2: Simulation the advection of passive tracers for a 2D model of a blood vessel with a large aneurysm. The flow was obtained by solving the Navier-Stokes equation, with realistic boundary conditions. From (Schelin et al., 2009).

The rest of this paper is organised as follows. In Sections 2 and 3, we introduce the concept of chaotic advection, and discuss the role of the chaotic saddle and its stable and unstable manifolds for the dynamics of open flows. In Sec. 4, we show how fractal distributions arise in chaotic open flows due to the chaotic saddle, and their consequences for the advection dynamics; and we introduce the concept of fractal dimension as a measure of the sensitivity to initial conditions and an indicator of strong mixing. In Section 5, the non-hyperbolic case is examined, and the consequences of KAM islands to mixing are explored. Section 6 takes molecular diffusion into account, and discusses what are the appropriate measures of the efficiency of mixing for real-world cases; the discussion is illustrated with experimental results in the context of industrial mixing. Finally, in Section 7 the application of these concepts to the dynamics of plankton in the ocean is presented.

\section{Example of an open flow: the blinking vortex-sink system}

We will now introduce a very simple open flow which exhibits chaotic advection and is given analytically as a mapping, which means that there is no need to solve Navier-Stokes equations to study and simulate it. We will 
use this flow to illustrate many of the concepts relevant to chaos and open flows in the following.
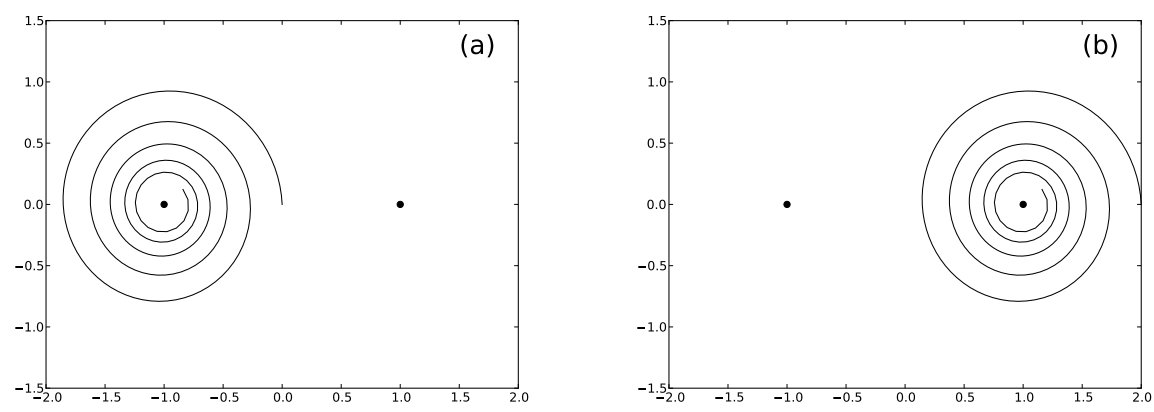

Figure 3: Illustration of the blinking-vortex flow. The sinks are indicated by the black circles. During the first half of a period, the left sink is active and flow spirals towards it (a), while during the second half half of the period, the left sink is closed and the right sink is open, as shown in (b). This switching from on sink to the other is iterated indefinitely.
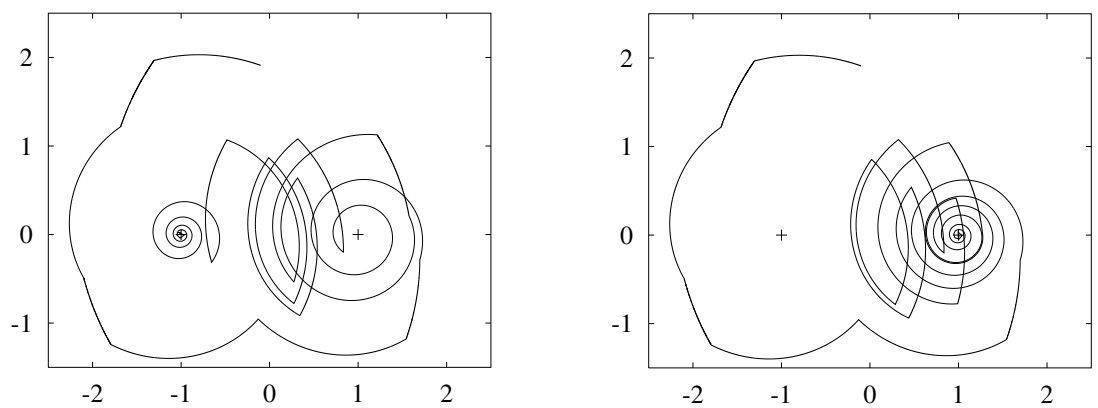

Figure 4: Trajectories corresponding to two very close initial conditions in the blinking vortex-sink flow. From (Tél et al., 2005).

The blinking vortex-sink flow (Aref et al., 1989; Károlyi and Tél, 1997) is a generalisation of Aref's blinking vortex flow (Aref, 1984). It is a 2D incompressible flow on an infinite plane, with two sinks which open and close periodically in alternation: in the first half of each cycle one sink is open 
and the other one is closed, and in the second half the situation is reversed (see Fig. 3). As fluid falls into the sink it spirals around it, and so each sink is also a vortex. Each vortex-sink is modelled as a point-source of vorticity superimposed to a localised sink, and we assume that the flow which falls on either of the sinks disappears from the system and does not come back. This is clearly an open flow, where the inflow region corresponds to the whole space beyond the sinks. The outflow region is not modelled explicitly: fluid particles are assumed to simply disappear when they fall into a sink. Being a two-dimensional incompressible flow, advection in the blinking vortex-sink system is Hamiltonian (see Sec. 3 below).

Consider first a single vortex-sink, in a coordinate system where the origin coincides with it. It generates a velocity field with a radial component $u_{r}=$ $-C / r$ corresponding to the sink, and a tangential component $u_{\varphi}=K / r$ modelling the spiralling motion of the fluid as it falls into the sink.

A fluid particle follows a trajectory determined by the following equations of motion:

$$
\dot{r}=-C / r, \quad \dot{\varphi}=u_{\varphi} / r=K / r^{2} .
$$

Solving these equations with initial conditions $r_{0}$ and $\varphi_{0}$, we get

$$
r(t)=\left(r_{0}^{2}-2 C t\right)^{1 / 2}, \quad \varphi(t)=\varphi_{0}-\frac{K}{C} \ln \frac{r(t)}{r_{0}} .
$$

To get the blinking vortex-sink system (Aref et al., 1989; Károlyi and Tél, 1997), we just need to put two vortex-sink points on the plane and then turn them on and off alternately for a duration $T / 2$ as explained above, thereby generating a time-periodic flow of period $T$. Without loss of generality, we choose the positions of the vortices at $x= \pm a, y=0$, where $a$ is a parameter of the system. Since we have an analytical expression for the motion of fluid particles for each of the half-periods, it is not difficult to find an expression for the new position $\mathbf{r}_{n+1}$ after one period as a function of the position $\mathbf{r}_{n}$ at the beginning of the period. This is best done using a complex representation for the position of a fluid particle, $z=x+i y$. The mapping from the initial position $z_{n}$ to the new one $z_{n+1}$ is then given by

$$
\begin{gathered}
z_{n+1 / 2}=\left(z_{n}+a\right)\left(1-\frac{C T}{\left|z_{n}+a\right|^{2}}\right)^{1 / 2-i K / 2 C}-a ; \\
z_{n+1}=\left(z_{n+1 / 2}-a\right)\left(1-\frac{C T}{\left|z_{n+1 / 2}-a\right|^{2}}\right)^{1 / 2-i K / 2 C}+a .
\end{gathered}
$$


Here $z_{n+1 / 2}$ is an intermediate variable representing the particle's position after the first half-period.

Dimensional analysis reveals that the dynamics is fully determined by the two dimensionless parameters:

$$
\eta=C T / a^{2}, \quad \text { and } \quad \xi=K / C,
$$

which can be interpreted as the dimensionless sink strength and the ratio of the vortex to sink strength, respectively.

Fluid particles escape from the system when they are too close to one of the vortex-sinks while it is active. It is easy to derive from Eq. (2) that the portions of the fluid which are within a distance of $R=\sqrt{C T}$ of a sink, at the instant when it starts to be active, will leave the system in the next half-cycle. Two different trajectories of fluid particles in this system are illustrated in Fig. 4.

\section{Chaotic advection in open flows}

Let us consider now an open flow of an incompressible fluid. A fluid particle at a given point $\mathbf{r}$ at time $t$ has a velocity given by the velocity field $\mathbf{u}(\mathbf{r}, t)$ of the flow at that point. The equation of motion for fluid particles is then

$$
\frac{d \mathbf{r}}{d t}=\mathbf{u}(\mathbf{r}(t), t)
$$

Finding an explicit expression for $\mathbf{u}(\mathbf{r}, t)$ involves solving the Navier-Stokes equation with the boundary conditions corresponding to the given physical system. It is usually not possible to solve the Navier-Stokes equation analytically in realistic scenarios, but here we will focus on the advection dynamics for a given flow field, and from now on we will simply assume that $\mathbf{u}(\mathbf{r}, t)$ is given. The results we present here are completely independent on how one goes about computing $\mathbf{u}(\mathbf{r}, t)$.

Equation (5) defines a time-dependent dynamical system with the number of degrees of freedom equal to the spatial dimension of the flow. This suggests the possibility that this dynamical system could be non-integrable, and displays chaos. From the point of view of the theory of dynamical systems, advection in open flows is a scattering process: there is a well-defined asymptotic region where the dynamics is simple - for example, in the flow past an obstacle, the fluid in the upstream and downstream regions moves at almost constant speed and direction; and there is a bounded region of 
space where the flow dynamics is complex - for example, in the wake of the obstacle the motion can be very erratic and unpredictable. This latter region is called the mixing region.

Another crucial feature of open flows is that the dynamics is transient: a typical fluid particle stays in the mixing region for some time after arriving from the upstream region, and then escapes again to the downstream region, never to return again. The type of chaos displayed by such systems is termed transient chaos (Lai and Tél, 2011); it is also sometimes called chaotic scattering. The subject of transient chaos is part of the fundamental theory of dynamical systems, and we can take advantage of the substantial body of results in this field to shed some light at the behaviour of chaotic open flows, and what the consequences of chaos are to mixing in this kind of flow.

The discussion of chaos in open flows becomes particularly simple in the case of two-dimensional incompressible flows, and we focus on this case for the remainder of this paper, unless otherwise noted. This is not only for the sake of simplifying our presentation: many very important systems can be modelled by 2D flows, including the Earth's oceans and atmosphere (Pedlosky, 1979). The incompressibility condition in 2D flows is expressed by

$$
\nabla \cdot \mathbf{u}=\frac{\partial u_{x}}{\partial x}+\frac{\partial u_{y}}{\partial y}=0 .
$$

This expression implies that there is a stream function $\Psi(x, y, t)$ which determines the flow's velocity (Landau and Lifshits, 2000; Batchelor, 1967):

$$
u_{x}(x, y, t)=\frac{\partial \Psi(x, y, t)}{\partial y} ; \quad u_{y}(x, y, t)=-\frac{\partial \Psi(x, y, t)}{\partial x} .
$$

Using the above expressions, the equation of motion for an advected particle can be written in terms of the stream function:

$$
\dot{x}=\frac{\partial \Psi}{\partial y} ; \quad \dot{y}=-\frac{\partial \Psi}{\partial x} .
$$

These two equations define a Hamiltonian dynamical system with one degree of freedom, where the variable $x$ plays the role of position, and $y$ plays the role of conjugate momentum, and the stream function $\Psi(x, y, t)$ is the Hamiltonian function (Landau and Lifshits, 2000; Batchelor, 1967). The phase-space of this Hamiltonian system is therefore the physical 2D space of the flow, and can be directly visualised in experiments by using dyes which are passive tracers (Sommerer, 1996). 
In stationary flows, the stream function is time-independent, and the fluid particle trajectories coincide with the streamlines, which are level curves of $\Psi$. In this case we have a time-independent Hamiltonian system with only one degree of freedom, and its dynamics is always integrable (that is, nonchaotic). This is similar to the case of a simple pendulum of one degree of freedom, whose dynamics is known from elementary classical mechanics to be always integrable. If the flow is non-stationary, however, the system is described by a time-dependent Hamiltonian, similar to that of a driven pendulum. It is well-known that such driven non-linear systems commonly have chaotic dynamics (Ott, 1993). In open systems this results in fluid particles having complicated, erratic motion for a transient period before they escape to the outflow.

Chaos is characterised by a sensitive dependence of the trajectories to small perturbations in the initial conditions. This is illustrated in Fig. 4 for the blinking sink-vortex system introduced earlier. Two initially very close trajectories eventually separate and leave to the outflow through different points, and at different times. This is a general property of chaotic systems: the same sensitivity to initial conditions is present in any chaotic flow. As an example, Fig. 5 shows the same phenomenon in a $2 \mathrm{D}$ flow past a cylindrical obstacle.

It is important to note here that even very simple time dependencies of the flow can give rise to chaotic advection. For example, time-periodic flows in general display chaotic advection (Aref, 1984; Ottino, 1989; Sommerer, 1996).

\section{Mixing in the presence of chaos: the chaotic saddle and the appearance of fractals}

Chaotic advection is characterised by the presence of a chaotic saddle, which is a set of orbits trapped in the mixing region. These are orbits that never escape the mixing region, and constitute an invariant set of the dynamics. A chaotic saddle has the property that none of its orbits escape the mixing region either in the forward dynamics $(t \rightarrow+\infty)$ or in the reverse dynamics $(t \rightarrow-\infty)$. There are infinitely many orbits in the chaotic saddle, including a countable set of periodic orbits of arbitrarily high periods, as well as an uncountable set of aperiodic orbits. These orbits are distributed in space in a fractal configuration, and this has a profound influence on the dynamics of advection. A snapshot of the chaotic saddle for the blinking 


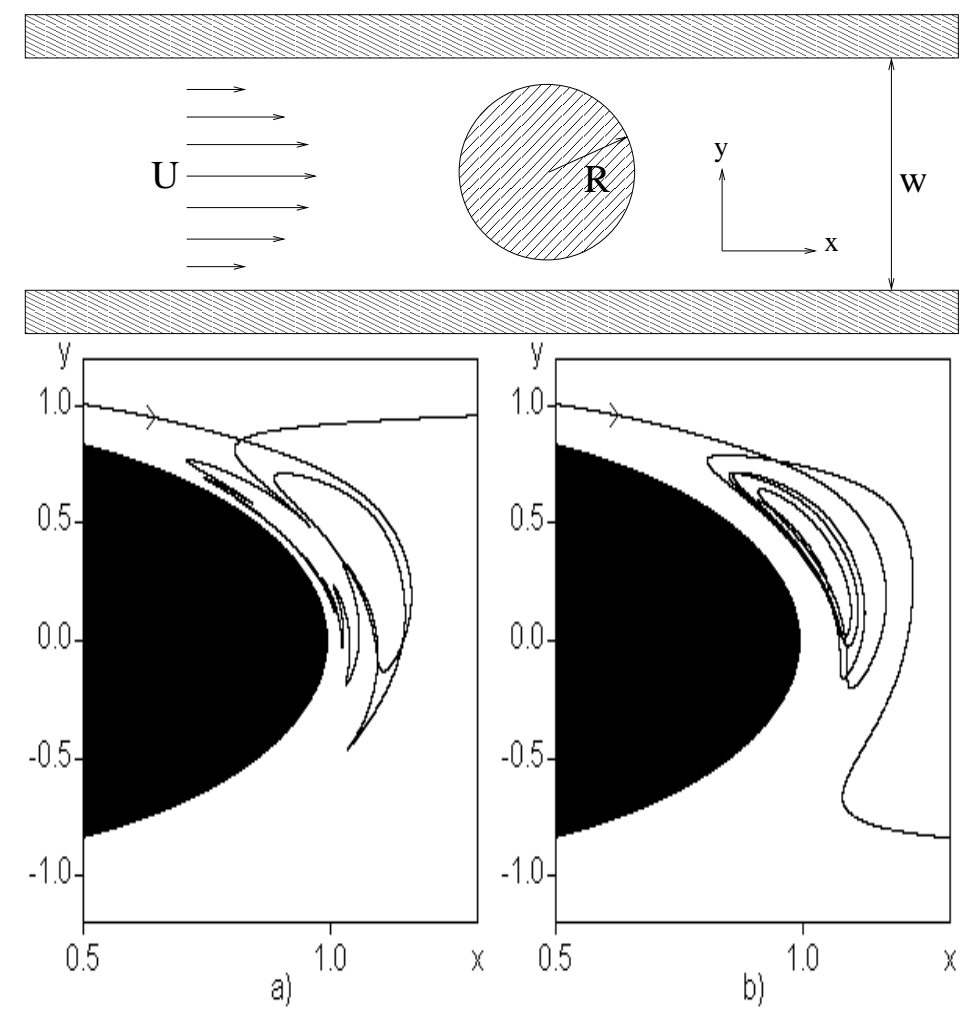

Figure 5: Illustration of a sensitivity of trajectories to initial conditions in a channel flow. Above: depiction of channel flow. Below: zoom-in on the region in the wake of the cylinder, showing the simulation of two trajectories corresponding to very close initial conditions. From (Tél et al., 2005).

vortex-sink flow is shown in Fig. 6. The self-similar structure characteristic of fractals is clearly seen.

The chaotic saddle has zero measure, which means that if we choose randomly points in a region of space, the probability of the chosen point being on the chaotic saddle is zero. In addition, the saddle is not an attractor: with probability 1 , orbits not exactly on the chaotic saddle will eventually escape to the outflow region. This is in contrast to strange attractors present in many nonlinear dissipative systems, where trajectories near the attracting set converge to the attractor. In this latter case it is easy to see why the 

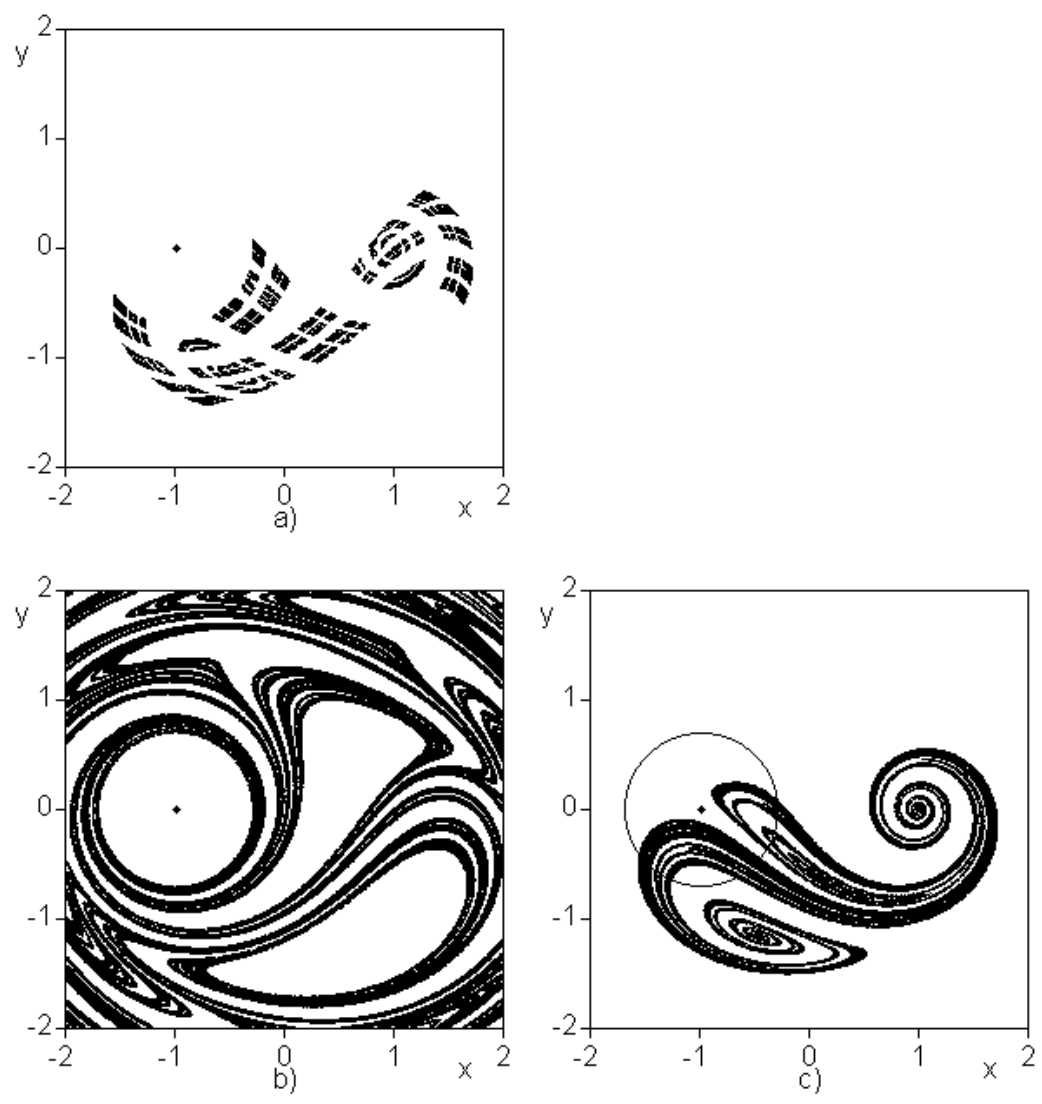

Figure 6: Snapshot of the a) chaotic saddle, b) stable manifold, and c) unstable manifold in the blinking vortex-sink flow $(\eta=0.5, \xi=10)$, taken at the beginning of each period. The circle in c) shows the area of the fluid that will escape during the next half time period. From (Tél et al., 2005).

fractal structure of the attractor is important for the dynamics. But the chaotic saddle is a non-attracting set, and although it is an invariant set of the dynamics, it might appear that since trajectories do not converge to it, it should not be important in practice. But nothing could be further from the truth: the chaotic saddle is the skeleton of a transient chaotic system, which determines the most important dynamical features of the system. In order to understand this, we must discuss the other invariant sets associated with the chaotic saddle. 


\subsection{The stable manifold of the chaotic saddle}

First let us consider the stable manifold of the chaotic saddle (we will refer to it as just the stable manifold, for conciseness). It is the set of initial conditions whose trajectories approach asymptotically the chaotic saddle, in the limit $t \rightarrow+\infty$. Fluid particles lying on the stable manifold enter the mixing region and never leave it, becoming 'trapped' there. However, the stable manifold has zero measure, like the chaotic saddle. This is a simple consequence of the incompressibility of the fluid: if a positive area of fluid belonged to the stable manifold, it would converge asymptotically to the chaotic saddle, which as we have seen has zero area; but this would violate the incompressibility property. As a result, almost all orbits started in the inflow region will leave the mixing region some finite time after entering it, and only a set of initial conditions of zero measure corresponds to trajectories which never leave.

The stable manifold for the blinking vortex-sink system is shown in Fig. 6 .

In spite of its zero measure, the stable manifold has a great influence on the global dynamics of the system. Orbits starting from points close to it will spend a long time in the interaction region before escaping, and these longlived trajectories are responsible for the sensitivity of the dynamics to initial conditions. To understand this point, consider initial conditions lying on a one-dimensional segment which crosses the stable manifold. Figure 7 shows the escape time of fluid particles with initial conditions on such a segment as a function of their positions (that is, how many periods it takes for a given fluid particle to escape through one of the vortex-sinks). At the points where the line intersects with the stable manifold, the escape time diverges, since the corresponding trajectories converge to the chaotic saddle and get trapped in the mixing region. Because the stable manifold is a fractal set, its intersections with the segment (and with any smooth curve) are infinite and non-enumerable, and constitute a Cantor set. This is the origin of the many peaks seen in Fig. 7, and also the origin of the wild appearance of the escape time function. Fig. 7 also shows a magnification of a small region of the plot, and we see that the plot does not become any smoother as we look closer. This is a consequence of the self-similar nature of the stable manifold: this intricate structure of peaks and troughs is found at all scales.

The sensitivity to the initial conditions can be understood as a direct result of the fractal nature of the stable manifold. Figure 7 shows that an observable quantity such as the escape time varies wildly in arbitrarily 

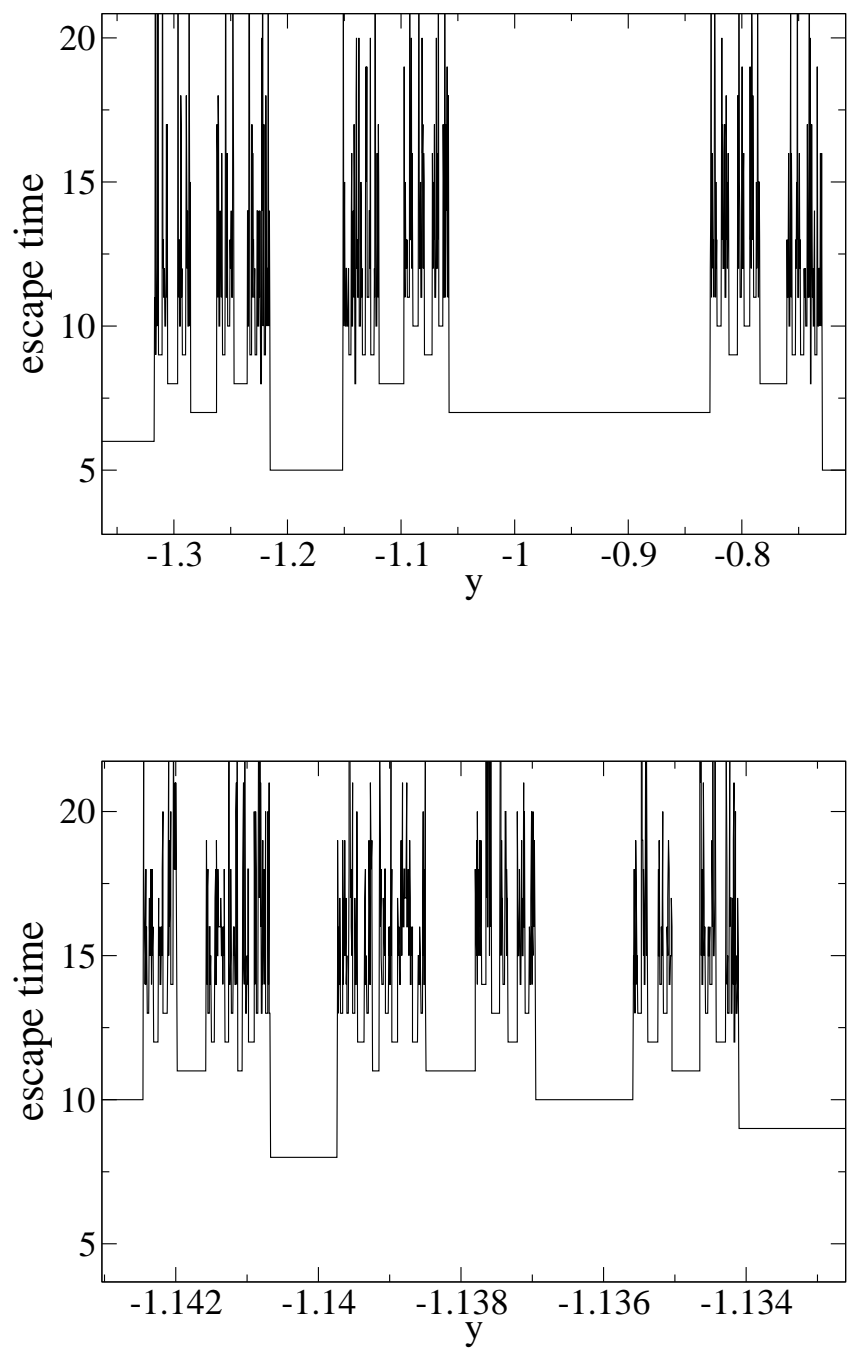

Figure 7: Plot of the escape time as a function of the initial condition in the blinking vortex-sink flow, for parameters $\eta=0.5, \xi=10$. In the top figure, the initial conditions are taken on the segment $x_{0}=0, y_{0} \in(-1.35,-0.7)$. The bottom figure is the magnification of a region of the top figure. 
small scales, and is therefore impossible to predict in regions close to the stable manifold. One way to understand this is considering that if there is a filament of the stable manifold lies between two fluid particles, they will end up following completely different trajectories, even if they started very close to each other; and since there is an uncountable infinity of these filaments, it is not difficult to see how the unpredictable and erratic behaviour of the trajectories near the stable manifold arises.

\subsection{The unstable manifold}

The other important set associated with the chaotic saddle is its unstable manifold. This is the set of points whose trajectories converge asymptotically to the chaotic saddle in the reverse dynamics, that is, as $t \rightarrow-\infty$. The unstable manifold is also a fractal set, composed of an intricate arrangement of filaments much like the stable manifold. Fig. 6 shows the unstable manifold for the blinking vortex-sink system. The physical meaning and relevance of the unstable manifold is due to the fact that those trajectories which stay a long time in the mixing region (that is, lying close to the stable manifold in the inflow region) will trace out the unstable manifold on their way out towards the outflow region. As a consequence, the unstable manifold can be observed directly in imaging experiments, by following a dye as it is advected (Sommerer, 1996). Once the bulk of the dye has escaped, what still remains in the observation region shadows the unstable manifold, and hence is distorted into a complex filamentary structure which becomes finer and finer as time passes and more dye is lost to the outflow. This process is illustrated in Fig. 8, and shown for the blinking vortex-sink flow in Fig. 9.

We remark that our discussion so far has focused on the motion of fluid particles, which coincides with the advection of passive scalars by the fluid in the absence of molecular diffusion. But the unstable manifold also manifests itself in the case of non-zero diffusion: as we will see in Sec. 6, the spatial concentration distribution of the scalar approaches a pattern which shadows the unstable manifold, with a lower length scale set by the diffusion and the mean stretching rate. Thus for small enough diffusion coefficients, the unstable manifold governs the long-time dynamics of the advection of scalars.

\subsection{The fractal dimension and its physical meaning}

The intricate fractal geometry of the invariant sets of chaotic flows, illustrated in Fig. 6 for the blinking vortex-sink flow, is intimately connected to the unpredictability of the dynamics and with the intensity of mixing in 

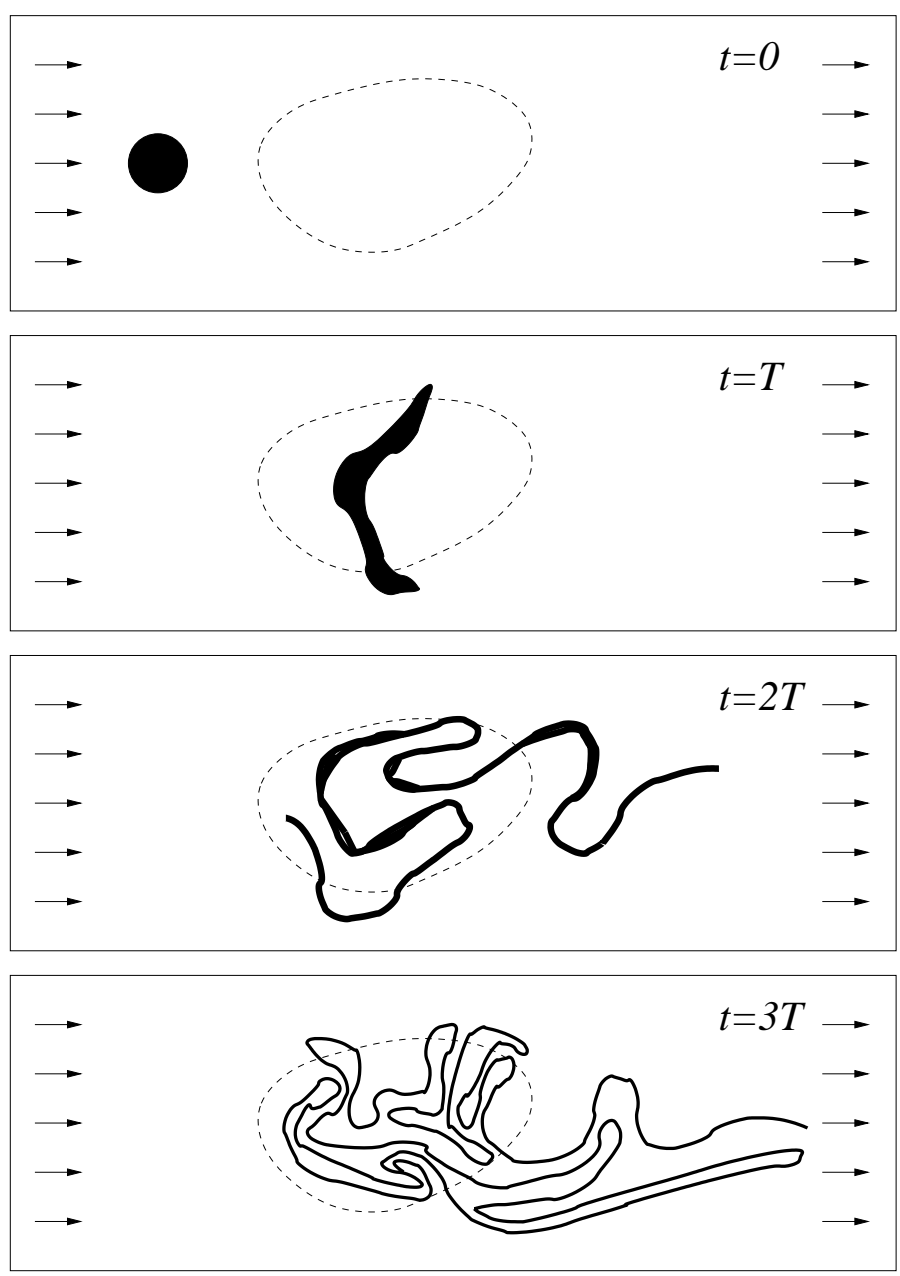

Figure 8: Illustration of a dye droplet reaching the mixing region of an open chaotic flow. After some time, the remaining dye traces out the unstable manifold of the chaotic saddle. From (Tél et al., 2005).

open flows. In order to quantify this relation, we review here the concept of fractal dimension (Halsey et al., 1986; Falconer, 2003), and we focus on its physical meaning and its connection to the dynamics of mixing.

Consider first the stable manifold of the chaotic saddle. It is composed of a fractal set of curves, as in Fig. 6. Imagine now a grid of resolution $\epsilon$ on a given region of interest, which intersects the stable manifold. Let $\mathcal{N}(\epsilon)$ be 

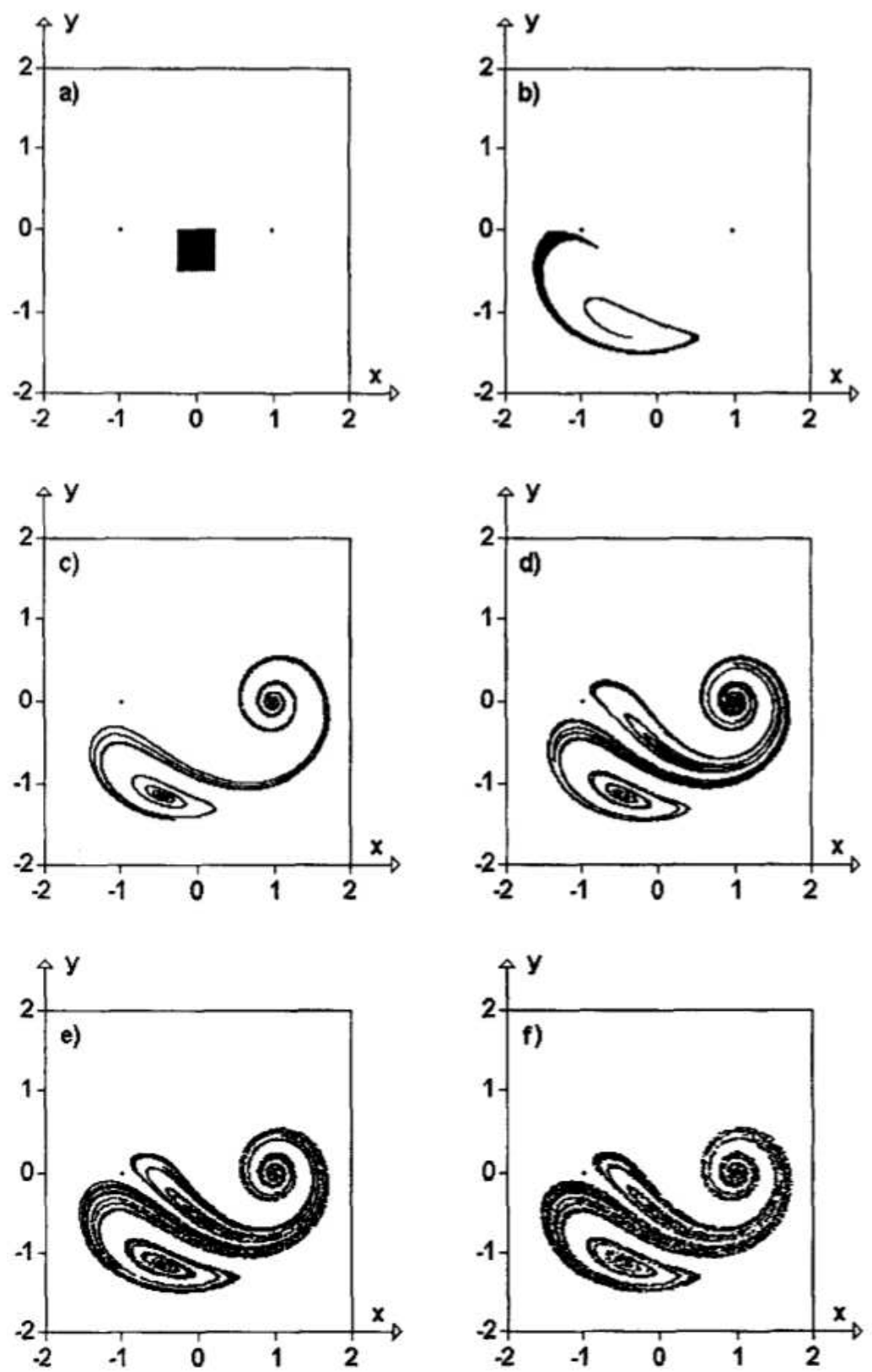

Figure 9: Evolution of a set of particles in the blinking vortex-sink flow. Compare the last picture with Fig. 6c. From (Tél et al., 2005). 
the number of grid cells containing points in the stable manifold. The fractal dimension $D$ of the stable manifold is defined from the way $\mathcal{N}$ scales with $\epsilon$. In general the scaling follows a power law: $\mathcal{N}(\epsilon) \sim \epsilon^{-D} ; D$ is defined as the fractal dimension. More precisely:

$$
D=\lim _{\epsilon \rightarrow 0} \frac{\ln \mathcal{N}(\epsilon)}{\ln (1 / \epsilon)} .
$$

For objects of regular geometry, $D$ corresponds to the usual notions of dimension: a one-dimensional curve has $D=1$, and a surface has $D=2$, for instance. But for fractal entities such as the invariant manifolds of chaotic flows, $D$ is generally not an integer. For the stable (and unstable) manifold, composed of a fractal set of curves, the fractal dimension satisfies $1<D \leq 2$ in two dimensions.

Equation (9) is in fact one of the many possible definitions of a fractal dimension; it is called the box-counting dimension, and also the capacity dimension. Since it is the only definition we will use in this work, we will refer to $D$ from now on as simply the fractal dimension.

One of the reasons why the fractal dimension is such an important concept is that it can be interpreted as a measure of the uncertainty of the dynamics of a transient chaotic system. In order to justify this interpretation, let us say we have an uncertainty $\delta$ in the determination of the initial condition of a particle of a passive tracer whose trajectory we want to predict. For example, we would like to be able to tell when the particle will leave the mixing region, and where it will be in the outflow region when it does leave. As discussed in Sec. 4.1, trajectories with initial conditions located in the vicinity of the stable manifold are extremely hard to predict. There is therefore an "uncertain region", consisting of the region in space within a distance of about $\delta$ from points in the stable manifold, wherein prediction is effectively impossible. A good measure of the uncertainty for the given accuracy $\delta$ is then the area $A(\delta)$ of the uncertainty region. From the definition of fractal dimension in Eq. (9), the number of "boxes" of size $\delta$ intersecting the stable manifold scales as $\delta^{-D}$, for sufficiently small $\delta$. Since each box has area $\delta^{2}$, the total area of the uncertain region scales as

$$
A(\delta) \sim \delta^{2-D} .
$$

The exponent $2-D$ determines how the size of the uncertainty region depends on the accuracy $\delta$. In non-chaotic flows, the stable manifold is a regular set 
of curves, with $D=1$. In this case, the uncertainty area is proportional to $\delta$, so that if we increase the accuracy by a factor of, say, 10, $A$ will decrease by the same factor. But in chaotic flows, $D>1 ; A$ then decreases more slowly with $\delta$, meaning that increases in accuracy have a much reduced effect on the area of the uncertainty region. This effect becomes extreme for values of $D$ close to 2 . For the case of $D=1.9$, for example, it would take a decrease of ten orders of magnitude in $\delta$ to reduce $A$ by a factor of 10 .

The area $A(\epsilon)$ is proportional to the probability of a randomly chosen initial condition, when perturbed to a nearby initial condition at a distance $\epsilon$ away, to be on the other side of one of the filaments of the stable manifold, resulting in very different outcomes for the two initial conditions. This observation suggests a way of measuring $D$, by picking a large number of randomly chosen pairs of points separated from each other by a distance $\epsilon$, and simulating their orbits, to ascertain if the two trajectories are similar (for example, by comparing their escape times); those pairs which do not have similar orbits are labelled "uncertain pairs". By the discussion above, the fraction $f(\epsilon)$ of uncertain pairs is proportional to $A(\epsilon)$. The function $f(\epsilon)$ can be found numerically by computing the fraction of uncertain pairs for several values of $\epsilon$ and plotting the result in a log-log plot; the slope of that plot gives the exponent $2-D$. More rigorously, the fractal dimension and the uncertain fraction are related by

$$
D=2-\lim _{\epsilon \rightarrow 0} \frac{\ln f(\epsilon)}{\ln \epsilon} .
$$

An example of this calculation is shown for the blinking vortex-sink system in Fig. 10.

Although the discussion above was centred on the stable manifold, by the time-reversal property of Hamiltonian systems, the unstable manifold has the same fractal dimension as the stable manifold. Since the trajectories which spend long times in the mixing region converge to the unstable manifold, this is where most of the mixing takes place. Therefore, the fractal dimension $D$ also measures the strength of the mixing in open flows: the greater the fractal dimension, the greater the amount of mixing happening in the flow.

\subsection{The Grassberger-Kantz relation}

Dynamical systems can be classified into hyperbolic or non-hyperbolic, depending on the stability properties of the orbits in their chaotic saddles. In hyperbolic systems, all orbits in the chaotic saddle are unstable. A hallmark 


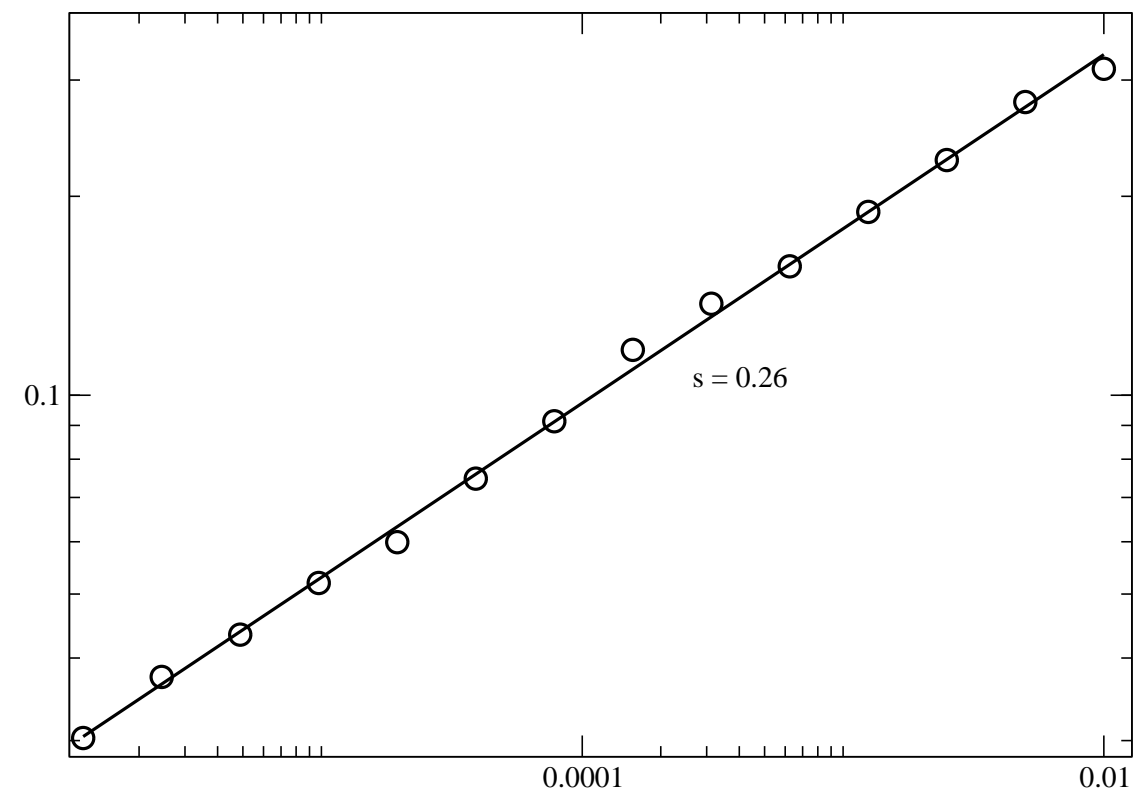

Figure 10: Numerical calculation of the uncertainty fraction $f(\epsilon)$ for the blinking vortex-sink system, with $\eta=0.5, \xi=10$. The fractal dimension is given from the slope $s$ by $D=2-s \approx 1.74$.

of hyperbolic systems is that they have an exponential decay: that is, if we keep track of the time evolution of a typical area of flow, the amount $Q(t)$ of this initial area still remaining in the mixing region at time $t$ decays exponentially with $t$ for large $t: Q(t) \sim e^{-\kappa t} . \kappa$ is the escape rate of the flow. It satisfies $\kappa<\lambda$, where $\lambda$ is the chaotic saddle's Lyapunov exponent. The physical meaning of the Lyapunov exponent is that it measures the mean exponential rate of separation of nearby fluid particles while they are in the mixing region. The fractal dimension $D$ of the unstable manifold, the Lyapunov exponent $\lambda$ and the escape rate $\kappa$ are related by the Grassberger- 
Kantz formula (Grassberger and Kantz, 1985):

$$
D=2-\frac{\kappa}{\lambda}
$$

More rigorously, we should have $D_{1}$, the information dimension (Falconer, 2003), instead of the box-counting $D$ in the expression above, but since $D$ and $D_{1}$ are almost always very close for open flows, this approximation is valid in most cases.

\subsection{Robustness of the chaotic saddle}

In all the discussion above, and in most of what follows, we concentrate on the case of two-dimensional flows. Furthermore, we have concentrated on the motion of fluid particles, that is, of passive tracers which assume exactly the velocity of the surrounding fluid. The fractal structure of the chaotic saddle and its associated invariant manifolds persist, however, in the case of actual, finite-sized particles, which have inertia and whose velocities do not coincide with that of the fluid's velocity field (Vilela et al., 2006, 2007; Cartwright et al., 2010). There are some considerable differences between the dynamics of fluid particles and that of inertial particles, in particular the possibility of the appearance of attractors in the latter case (Benczik et al., 2002; Motter et al., 2003; Cartwright et al., 2010). But even when the global dynamics has attractors, chaotic saddles are still present, and the system is still governed by fractal structures in phase space connected to a chaotic saddle, as in the simpler case of passive advection.

The same overall picture remains valid for three-dimensional systems (Cartwright et al., 1996; Tuval et al., 2004; de Moura and Grebogi, 2004a); in this case, the stable and unstable manifolds are a fractal set of sheets, instead of segments. Periodicity is also not required for the existence of the chaotic saddle: aperiodic and random flows can also result in well-defined fractal structures in phase space (Károlyi et al., 2004; Rodrigues et al., 2010).

A conclusion of the above discussion is that the concepts of chaotic saddle and its stable and unstable manifolds are remarkably robust, and are not consequences of over-simplified models of flows. As a result, we expect fractal sets to be features of real flows which are dominated by large-scale coherent structures; if the flow is dominated by well-developed turbulence, on the other hand, this is no longer true. 


\section{Transport barriers and KAM islands: the effective dimension}

In discussions about chaotic open flows and the chaotic saddle it is often assumed, sometimes tacitly, that the dynamics is hyperbolic. The reason is partly that the hyperbolic case is more treatable, and there are more rigorous results available about that case. For example, the Grassberger-Kantz relation (12) is only valid for hyperbolic systems. However, non-hyperbolicity occurs in many important cases, and is to be expected in many very general scenarios in fluid dynamics. For example, it can be shown that the dynamics of $2 \mathrm{D}$ advection on a flow past an obstacle becomes chaotic immediately after the transition of the flow from stationary to time-dependent, as the Reynolds number is increased beyond a critical value; furthermore, the dynamics is non-hyperbolic for a range of Reynolds numbers past the transition point, independently of the shape of the obstacle or the particular features of the flow (Biemond et al., 2008). Many other systems of interest are nonhyperbolic, and it is imperative that we understand the mixing dynamics in the non-hyperbolic case.

Non-hyperbolicity is manifested through the appearance of stable orbits in space. These orbits are surrounded by stable islands, from which fluid does not escape. These stable regions correspond to persistent vortices in the flow. In dynamical systems parlance, they are KAM islands (MacKay and Meiss, 1987). KAM vortices are commonly found in 2D flows, and they have been observed in environmental flows, such as the stratospheric polar vortex, which plays a crucial role in the process of ozone depletion (Koh and Legras, 2002); and also in ocean circulation patterns (Abraham, 1998; Boyd et al., 2000; Abraham et al., 2000). As is well known from the theory of Hamiltonian dynamical systems, they form a fractal hierarchical structure, with big islands being surrounded by smaller islands, and these in turn are surrounded by even smaller islands, and so on (see Fig. 11). The presence of KAM islands means that there is a finite volume of initial conditions in the mixing region whose orbits do not escape, corresponding to those initial conditions lying in the islands. However, fluid particles with initial conditions outside the interaction region cannot enter the islands. As a result, the set of initial conditions outside the mixing region whose trajectories end up trapped there still has zero measure, as in the hyperbolic case. However, the islands have deep consequences for the scattering dynamics, resulting in important differences between the hyperbolic and non-hyperbolic cases.

The transport of fluid in the vicinity of the islands is dominated by Can- 


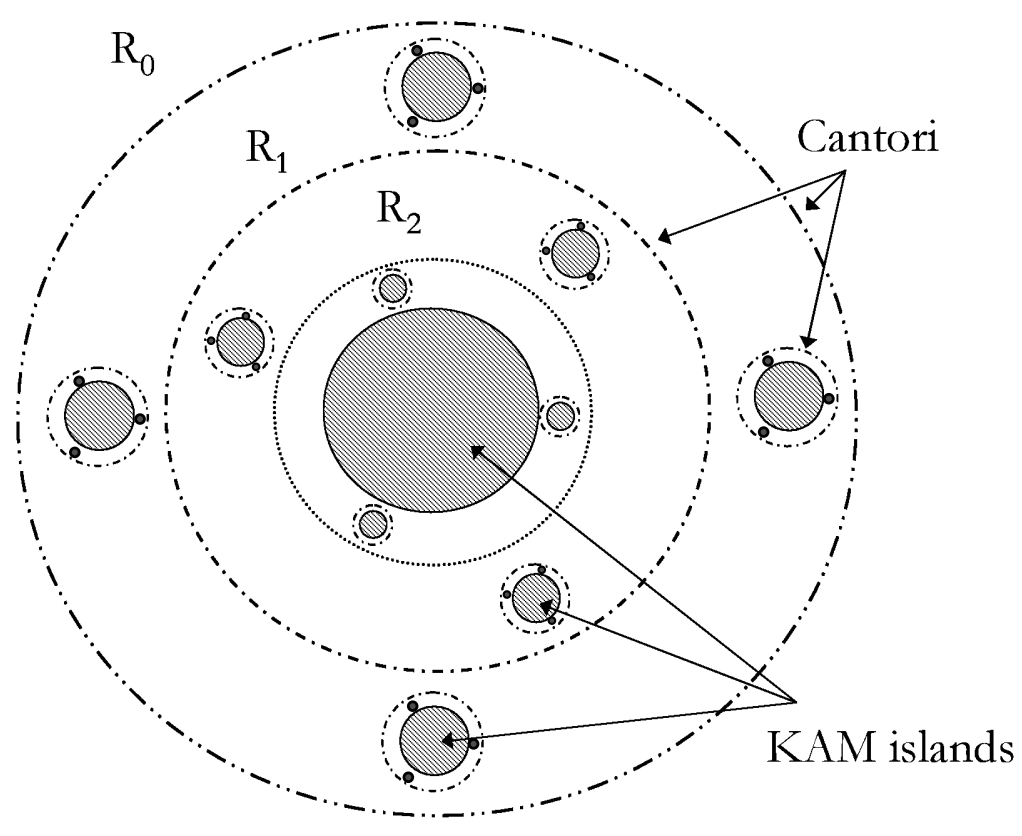

Figure 11: Illustration of the hierarchical structure of KAM islands and Cantori. Solid circles represent KAM tori, and Cantori are represented by the circles with holes. From (Tél et al., 2005).

tori, which are remnants of broken up KAM tori. Cantori are also invariant sets of the dynamics, as are KAM islands; but in contrast with those, fluid particles can cross from one side of a Cantorus to the other. However, it takes typically very long times to do so, and as a consequence the Cantori act as partial transport barriers. The overall picture of non-hyperbolic transport is sketched in Figure 11.

As we mentioned above, it is very common to find KAM islands in fluid flows, the blinking sink-vortex flow is no exception. Figure 12 shows stroboscopic trajectories for a set of parameters for which the flow is non-hyperbolic, and an island is clearly visible. The magnification in Fig. 12 shows the striking self-similar organisation of the islands. The effect of Cantori on the advection dynamics can be seen in the cloud of points surrounding the sub- 
islands on the upper right and to the left of the main island in the magnified figure. These points are snapshots taken at the start of every period of a single orbit which meanders inside the Cantorus surrounding these islands. This orbit eventually escapes, after thousands of cycles. Another Cantorus can just be seen surrounding the main island. These Cantori are in turn surrounded by a bigger Cantorus encircling the whole structure, which is apparent from the higher density of points in the region around the complex of islands in the bottom figure 12 .

An example of KAM islands visualised in an actual experiment is seen in Fig. 22.

\subsection{Dynamical consequences of non-hyperbolicity}

The partition of space by the KAM islands and Cantori into distinct domains separated by transport barriers has no counterpart in hyperbolic systems, and is the cause of the profound differences in the dynamics of hyperbolic and non-hyperbolic flows. A direct consequence of the self-similar structure of the transport barriers depicted in Fig. 11 is the phenomenon known as stickiness: in non-hyperbolic flows, many trajectories spend extremely long times inside Cantori, leading to very long typical escape times compared to hyperbolic dynamics. Because of the self-similar organisation of the Cantori, once inside, an orbit may enter an inner Cantorus located within another Cantorus, and so on to arbitrarily high levels in the Cantorus hierarchy. So once a fluid particle is inside a Cantorus, it will wander within a fractal labyrinth from which escape is likely to take a very long time.

Even in non-hyperbolic flows it is still true that fluid particles with initial conditions outside of KAM islands will eventually escape with $100 \%$ probability: the component of the chaotic saddle outside the islands has zero measure. But stickiness makes escape sub-exponential, in marked contrast with hyperbolic flows, where escape is exponential. In non-hyperbolic flows, the number $N(t)$ of particles, with initial conditions chosen randomly in a region with no intersection with KAM islands, that have not escaped up to time $t$, follows a power law (Meiss and Ott, 1985):

$$
N(t) \sim t^{-\gamma}
$$

with $\gamma>0$.

A direct consequence of the slower escape dynamics described by Eq. (13) is that the fractal dimension $D$ of the stable (and unstable) manifold is equal 

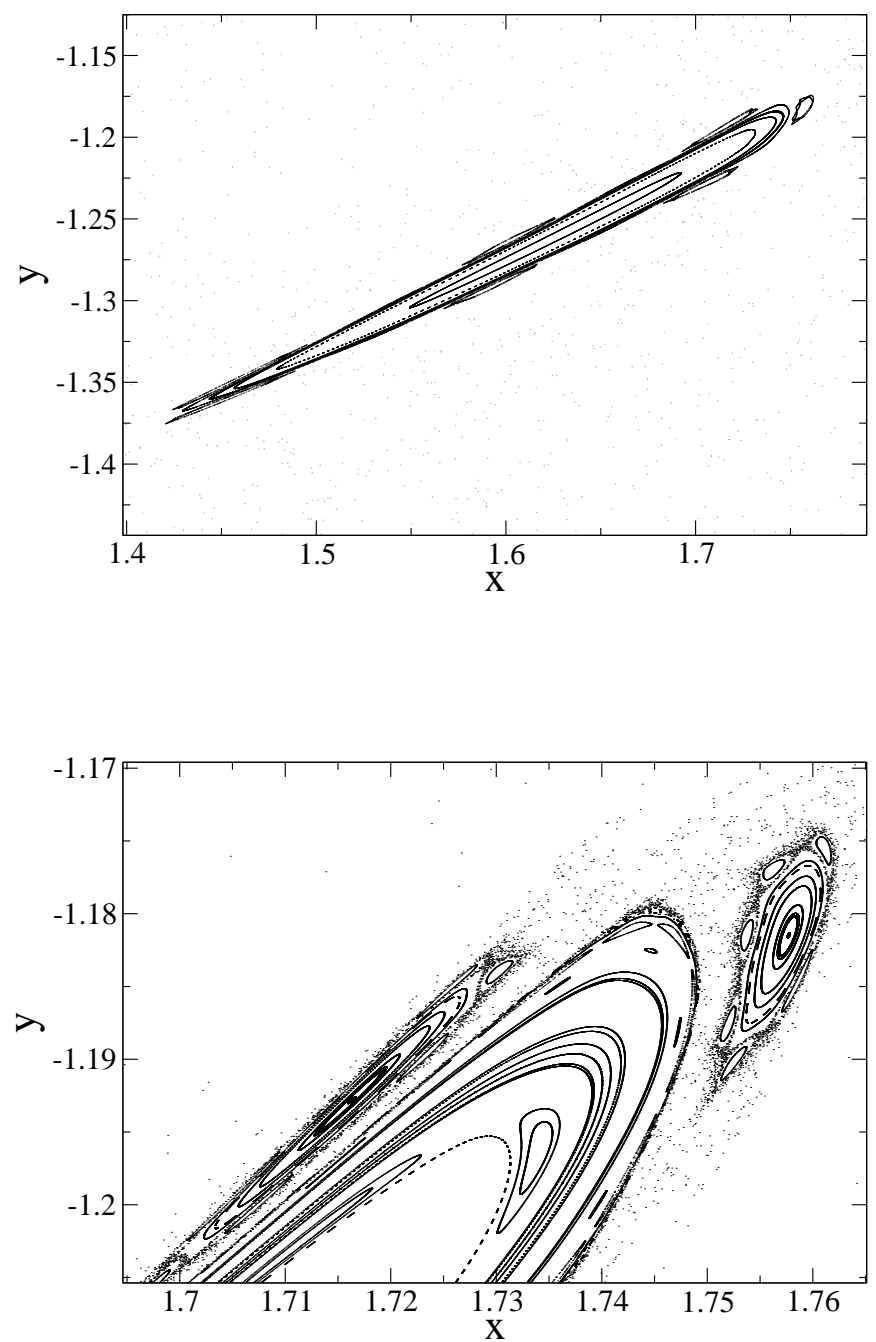

Figure 12: Stroboscopic map of the blinking vortex-sink flow, with $\eta=1$, $\xi=18$, parameters for which the flow is non-hyperbolic. The map shows the orbits of a few fluid particles, with positions taken at discrete times, at the beginning of every period of the flow. The picture on the bottom is a magnification of a small region of the top picture, and shows the self-similar structure of the KAM islands. 
to the dimension of the embedding space, $D=2$ (Lau et al., 1991). This is not inconsistent with the fact that the stable and unstable manifolds have zero measure, since fractal sets can have fractal dimension equal to the dimension of the phase space and still have zero measure (Falconer, 2003); and this is indeed the case of the stable and unstable manifold of non-hyperbolic transient systems. However, from the interpretation of the fractal dimension as a measure of uncertainty of transient systems, expressed mathematically by Eq. (11), the fact that $D$ assumes the maximum possible value in nonhyperbolic systems suggests that these systems have an extreme sensitivity to initial conditions. Indeed, the exponent in Eq. (11) vanishes for $D=2$, which means that the "uncertainty probability" $f(\epsilon)$ decreases more slowly than a power law for small $\epsilon$.

This extreme sensitivity of the dynamics is apparent from the plot of the escape time as a function of the initial conditions. This is shown in Fig. 13, with initial conditions taken in the interior of the outermost Cantorus of Fig. 12. The escape time is a very irregular function, with wild oscillations nearly everywhere; and the magnification shows that this irregular behaviour is present down to arbitrarily small scales.

\subsection{The effective dimension}

A glimpse at Fig. 13 suggests that predicting asymptotic properties of trajectories in this system is an almost impossible task. The reason for this unpredictability is the very long time it takes initial conditions inside Cantori to escape: two initially very close trajectories will have much more time to spend in the mixing region to separate and follow independent paths before they escape. Figs. 11 and 12 also suggest that the unpredictability is greater for initial conditions located in deeper levels of the Cantorus hierarchy, as they have longer escape times. To measure the uncertainty, we compute the uncertainty plot $f(\epsilon)$, as done in Sec. 4.3, using initial conditions inside the outermost Cantorus, and inside the inner Cantorus indicated by the cloud of points in the left plot of Fig. 12. Fig. 14. shows the result of this calculation for initial conditions picked in two different regions: inside the outermost Cantorus, and inside one of the inner Cantori seen in Fig. (12).

Figure 14 may seem to contradict the assertion made in Sec. 5.1 that $D=2$ for non-hyperbolic systems, as this would predict that the plot of $f(\epsilon)$ versus $\epsilon$ should be a flat line with zero slope. But in non-hyperbolic systems, the $\epsilon \rightarrow 0$ limit in Eq. (11) converges extremely slowly. For extremely small 

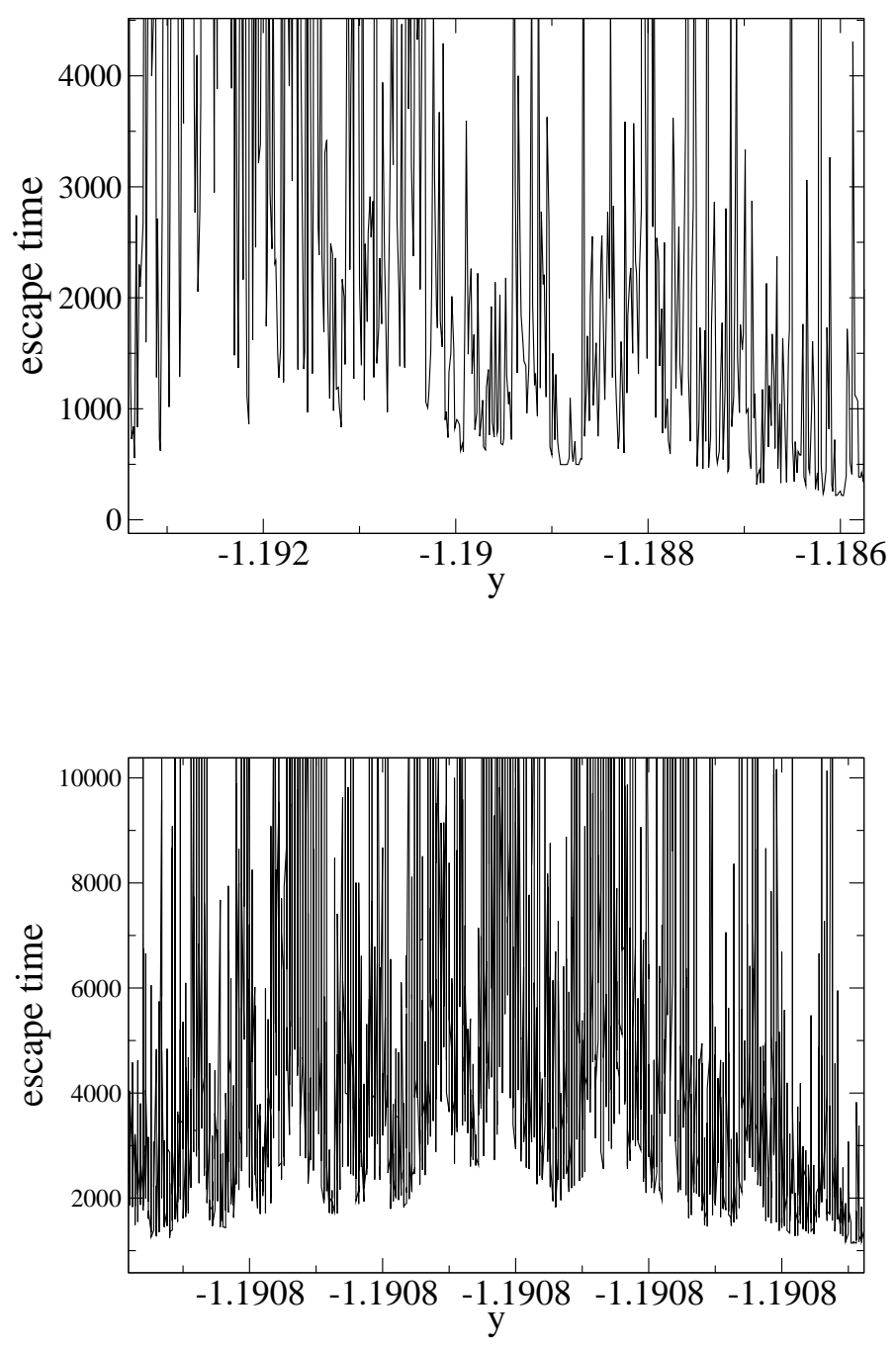

Figure 13: Escape time plot for the blinking vortex-sink flow, with $\eta=1$, $\xi=18$. Initial conditions are taken on a segment with $x_{0}=1.75$.

values of $\epsilon$, the slope does in fact approach 0 for $\epsilon$ small enough; but reaching this limit usually requires values of $\epsilon$ so small they are not physically relevant. Any model of a physical system has a lower scale below which the 


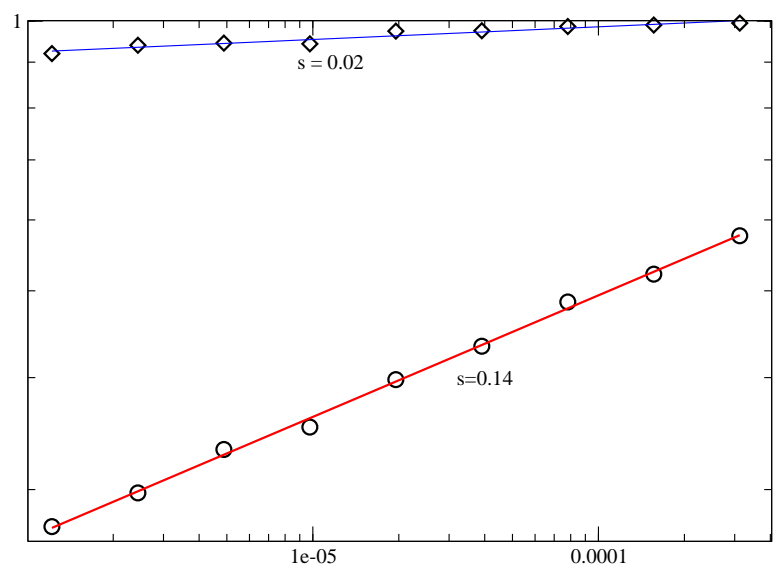

Figure 14: Fraction of uncertain pairs as a function of separation $\epsilon$ between points in a pair for the blinking vortex-sink flow, with $\eta=1, \xi=18$; see Section 4.3. Initial conditions are for the bottom curve are taken in the outermost Cantorus, on the segment $x_{0}=1.75, y_{0} \in(-1.18,-1.175)$; for the top curve, initial conditions are in an inner Cantorus, on $x_{0}=1.75, y_{0} \in$ $(-1.193,-1.192)$. The numbers beside each curve are the slope coefficients obtained from fitting, $f(\epsilon) \sim \epsilon^{s}$.

model is no longer valid; this may be given, for example, by the size of advected particles we are following in an experiment, of by the finite resolution of our measurements. This implies that the dimension that is relevant in realistic systems is not the mathematical definition (11) with its unreachable limit, but is given instead by an effective dimension $D_{\text {eff }}$ (de Moura and Grebogi, 2004b; Motter et al., 2005), defined as an approximation of the fractal dimension for a finite range of $\epsilon$ (see Fig. 15):

$$
D_{\mathrm{eff}}(\epsilon)=2-\frac{d \ln f(\epsilon)}{d \ln \epsilon} \approx \text { const. for } \epsilon_{1}<\epsilon<\epsilon_{2},
$$

valid in a range $\left(\epsilon_{1}, \epsilon_{2}\right)$, with $\epsilon_{1} \ll \epsilon_{2}$. $D_{\text {eff }}$ satisfies $D_{\text {eff }}(\epsilon) \rightarrow 2$ as $\epsilon \rightarrow 0$, in accordance with Eq. (11).

From Eq. (14), the results in Fig. 14 can be interpreted as yielding 


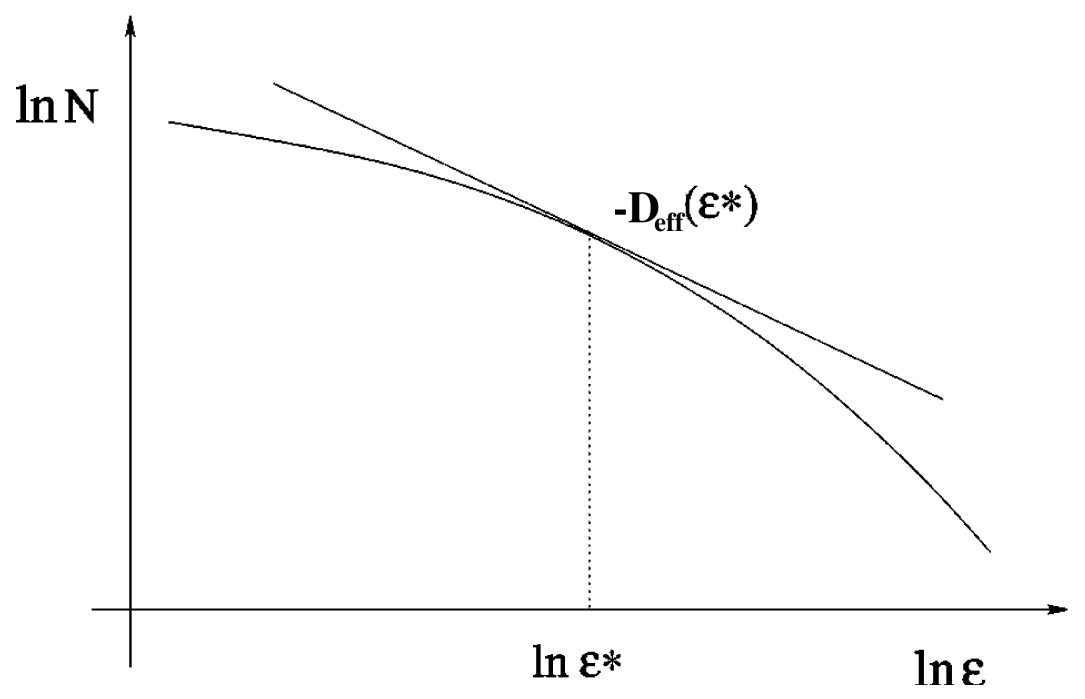

Figure 15: Illustration of the concept of effective dimension, the slope of the tangent to the $\ln N$ vs. $\ln 1 / \varepsilon$ curve.

the effective fractal dimension of the stable and unstable manifold for two different locations in space: $D_{\text {eff }}=1.86$ inside the outermost Cantorus, and $D_{\text {eff }}=1.98$ inside one of the inner Cantori. The effective dimension therefore depends on the position in non-hyperbolic systems, in contrast to the actual fractal dimension, which is 2 anywhere. The greater escape time in inner Cantori means that the invariant manifolds of the chaotic saddle have "more time" to be stretched and folded and distorted by advection, hence the greater effective dimension.

Because of time-reversal symmetry, the stable and unstable manifolds have the same fractal dimensions - and also the same effective fractal dimensions. We argued in Section 4.3 that the fractal dimension of the unstable manifold is a measure of lower-scale mixing efficiency for open flows. This means that the fluid in regions of space surrounded by Cantori will be extremely well-mixed, and the efficiency of mixing increases as we go deeper into the Cantorus structure, and reaches the maximum limit of $D_{\text {eff }} \rightarrow 2$ for regions buried deep within the Cantori. This picture is somewhat at odds with the prevalent idea in much of the literature of this field that the presence of KAM islands is an impediment to mixing. This assertion is valid in closed flows, for which optimal mixing requires mixing the fluid homoge- 
neously throughout the container; this is obviously not possible with KAM islands. In open flows, however, the fluid to be mixed usually comes from the inflow region, and thus from outside the KAM islands, and so this is not a problem. Instead, the islands cause fluid to spend very long times within the Cantori, and be very well mixed as a result. Therefore, in contrast to closed flows, in open flows KAM islands can be said to enhance mixing. However, it is important to note that our measure of mixing only takes into account the small-scale limit, and it ignores molecular diffusion. It also ignores the time it takes to achieve good mixing, since the stable and unstable manifold are all defined in the asymptotic dynamics. In industrial applications and other cases of interest all these factors must be taken into account, and other, more practical definitions of mixing efficiency are desirable. These will be introduced in Section 6.

\section{Mixing fluids by stirring in continuous-flow processes}

In the previous sections we focused on the purely advective aspects of mixing, ignoring molecular diffusion entirely. In this Section, we take molecular diffusion into account, and investigate how diffusion in an open flow is affected by its chaotic dynamics, and what consequences this has for the efficiency of mixing. We will also revisit the definition of "good" mixing, taking diffusion into account.

\subsection{What does good mixing mean for a diffusive dye in an open flow?}

How are different fluids mixed together and homogenised in industrial continuous-flow devices? The elaboration of pulp and paper, cosmetics or processed food often involves a mixing step which is part of an integrated continuous-flow system of reactors (Paul et al., 2003). Continuous-flow manufacturing allows reducing waiting times and the handling of materials. At a smaller scale, some microfluidics devices also integrate open-flow micromixers (Lee et al., 2001; Stroock et al., 2002; Okkels and Tabeling, 2004) in labs-on-a-chip. For all these applications, it is crucial both to understand the physical mechanisms at play, and to use relevant measures of mixing quality (Danckwerts, 1952; Bryant, 1977; Ehrfeld et al., 1999; Aubin et al., 2003; Kukukova et al., 2009).

In such systems, inhomogeneous fluid enters the mixing reactor, and fluid that is continuously flowing out of the reactor has to satisfy some desired property - such as sufficient chemical homogeneity, a maximal concentration 
in some chemical species, a target rheology, etc.. An example of open-flow reactor is shown in Fig. 16. It consists in a free-surface channel of shallow rectangular section, where viscous fluid (cane-sugar syrup) flows continuously at a fixed flow rate. Fluid particles cross a mixing region, where two rod-stirrers (circled in blue in Fig. 16 (a)) move on intersecting trajectories (dashed lines in Fig. 16 (a)) and stretch passing fluid particles thanks to the chaotic advection. The experimental apparatus has been described elsewhere (Gouillart et al., 2009). In the following, we make use of experiments realised with this rod-stirring protocol to explain how a diffusive dye is mixed in open flows; the mechanisms presented here apply nevertheless to the wider class of open flows with a mixing region with a limited spatial extent.

In the experiment of Fig. 16, fluid inflowing into the mixing region is not homogeneous, as a blob of black dye contrasts with the surrounding dyefree fluid (Fig. 16 a)). In industrial systems also, the composition of the inflowing fluid is usually heterogeneous; it may for example consist in two parallel streams of different liquids, or in a succession of patches of different liquids that have to be blended together. The outflowing fluid, however, is required to be "mixed". Let us first examine the different snapshots in Fig. 16 in order to specify what this somewhat vague term of mixed encompasses. In contrast to the segregated state in the inflow, where black (dyed) and white (dye-free) fluid are well separated, the mixed state can be defined as a dye concentration pattern where all white fluid particles are close to black fluid particles, and vice versa. The snapshot of Fig. 16 b) has been taken a few rotation periods of the rods after the arrival of the blob inside the mixing region shown in Fig. 16 a). Filaments of dyed and dye-free fluid that flow out of the mixing region in the upper part of Fig. 16 b) are better mixed than the fluid inflowing in Fig. 16 a), because the filaments are thinner than the patches in Fig. 16 a). However, particles inside the white and black filaments are still surrounded by fluids particles that all have the same colour, as in the initial patches in the inflow. This means that the dye concentration levels are the same as in the inflow (that is, the initial concentration of the blob of dye, and the null concentration). This may be a problem if, for example, the application requires that there is a maximal acceptable concentration of one species (here, the dye) in the final product. In the snapshot of Fig. $16 \mathrm{c}$ ) that was taken a few stirring periods after b), dye particles look much better mixed than in Fig. $16 \mathrm{~b}$ ), because dye filaments were smeared out by molecular diffusion with dye-free fluid, resulting in intermediate grey concentration levels. Molecular diffusion ensures that the 


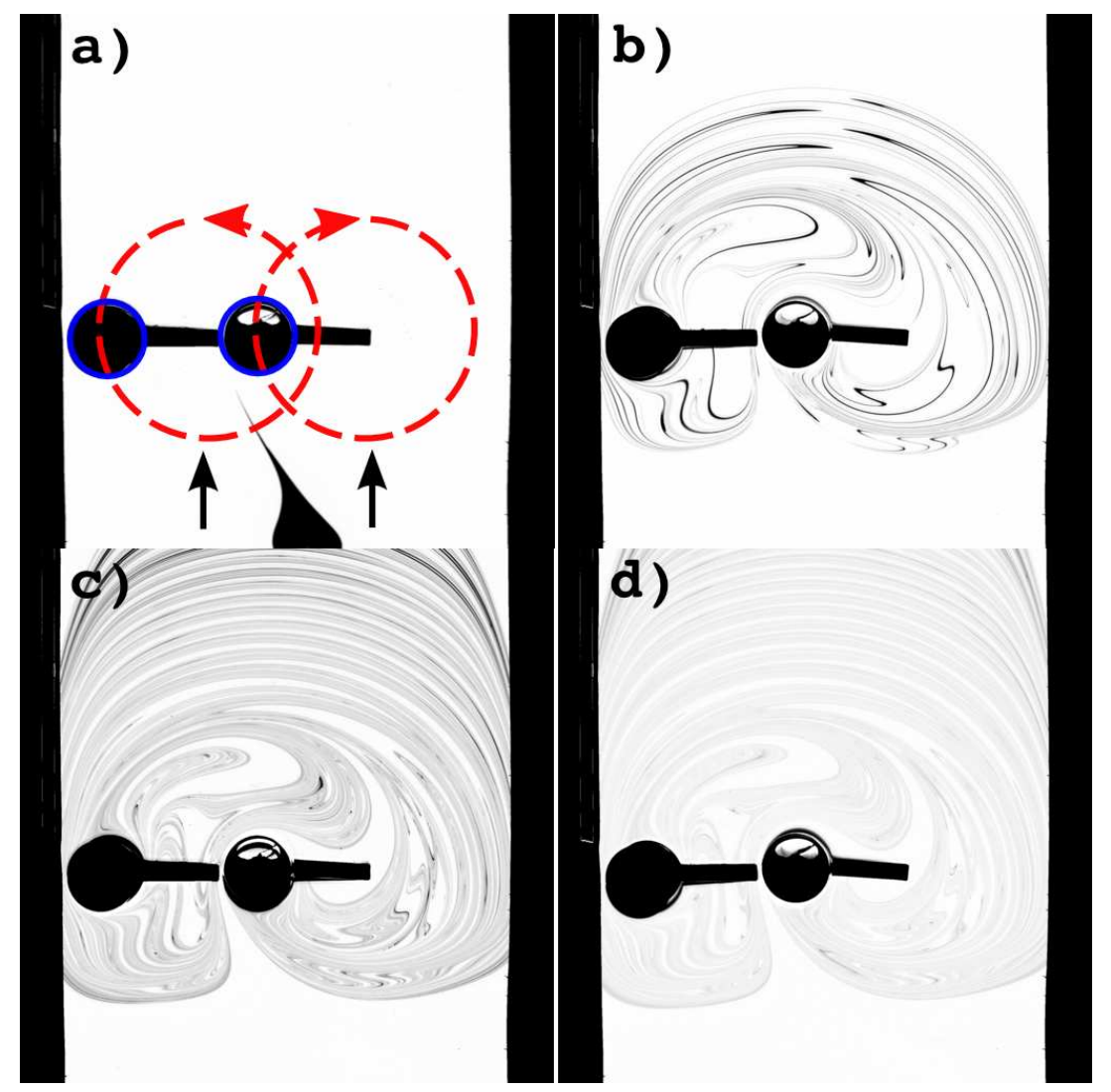

Figure 16: Snapshots of a blob of dye passing through an open-flow mixer, taken at different times. The main open flow is the upward direction. Two rods (circled in blue in a)) stretch and fold passing fluid particles. (a) Arrival of the dye blob in the mixing region (b) Three rotation periods of the rods after the entry of the blob (a)), unmixed dark filaments are flowing out of the mixing region, while the remainder of the initial blob keeps on being stretched by the rods. (c) Eight rotation periods after a), dye filaments inside the mixing region have been stretched enough to diffuse with dye-less fluid; hence fluid flowing out of the mixing region is well mixed. (d) Later on, the same pattern as in c) repeats, with the intensity of the dye that decays because of the open flow. 


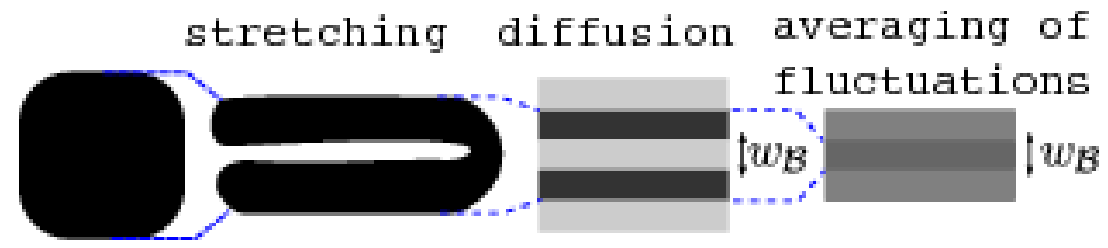

Figure 17: Schematic time-evolution of a patch of diffusive dye inside the mixing region. The patch is stretched and folded into many thin filaments by chaotic advection. Molecular diffusion starts to be efficient once the width of dye filaments reaches the Batchelor scale $w_{\mathcal{B}}$, at which the effects of chaotic stretching and diffusion balance. Dye filaments then diffuse with the surrounding fluid, and the contrast of the dye becomes weaker with time.

proximity between fluid particles, that were in different regions of space in the inflow, is realised at the molecular scale. In the following, we thus define good mixing for a patch of fluid as a state where all its fluid particles have been smeared out by molecular diffusion with fluid particles coming from different regions, that were bearing different concentration levels in the inflow. As this definition depends on the distribution of dye and inhomogeneities in the inflow, one may require for more generality that a fluid particle diffuses with other fluid particles that enter the mixing region at a different period of rotation of the rods.

How does an open-flow mixer such as the device in Fig. 16 achieve the diffusion-induced smearing of different dye concentration levels that is visible in Fig. $16 \mathrm{c}$ ) and d)? In fluid at rest, the timescale necessary for diffusion to be efficient at the scale of the reactor width $\ell$ is $\ell^{2} / \kappa_{\text {diff }}$, with $\kappa_{\text {diff }}$ the molecular diffusivity. As $\kappa_{\text {diff }}$ takes very small values in liquids $\left(10^{-8}-10^{-10} \mathrm{~m}^{2} . \mathrm{s}^{-1}\right.$ in water, and even lower values in more viscous fluids), such timescales are unrealistic for mixing in industrial large-scale installations. On the other hand, shears induced by a flow greatly increase the effective diffusivity. A famous example of this phenomenon is Taylor dispersion (Taylor, 1953). Taylor showed that a Poiseuille flow through a pipe increases molecular diffusion in the direction of the flow, because the shear increases the rate at which concentration gradients are smeared out by diffusion. However, this effect of a simple shear is too weak for most large-scale practical applications, and is inefficient in the direction transverse to the flow.

Chaotic advection achieves a more efficient enhancement of diffusion. The effect of chaotic advection on a patch of a diffusive dye is sketched in Fig. 17. 
Inside the mixing region where chaotic advection is at play, a patch of dye is elongated into filaments that get thinner and thinner with time as they are stretched more and more. After a short while, filaments reach the so-called Batchelor scale of the flow (Batchelor, 1959), at which diffusion starts to be efficient. The value of the Batchelor scale is given by

$$
w_{\mathcal{B}}=\sqrt{\frac{\kappa_{\text {diff }}}{\lambda}}
$$

with $\lambda$ the Lyapunov exponent introduced before, which is the mean stretching rate inside the mixing region. Dark and white filaments therefore start to diffuse into neighbouring filaments as it is sketched in Fig. 17, and concentration gradients are smeared out. The Batchelor scale corresponds to the scale at which the effects of diffusion and stretching balance, (Villermaux and Duplat, 2003) so that the width of dye filaments is stabilised at this scale, whereas they are smeared out with other filaments that are compressed into the same "box" of width the Batchelor scale (Fig. 17). The Batchelor scale is therefore a diffusive cut-off scale, which is the smallest length scale that can be observed in mixing patterns. This scale corresponds to the width of the thinnest filaments in Fig. 16 b-d.

With this understanding of the interplay between diffusion and chaotic advection, the definition of good mixing can now be specified as follows: a patch of dye is well mixed if it stretched enough by chaotic advection so that all the resulting filaments reach the Batchelor scale and are smeared out by diffusion (as in Fig. 17).

The above definition of good mixing applies for mixing in closed flows as well. In open flows, however, an additional challenge comes from the transient nature to chaotic advection, and the variety of residence times of fluid particles in the mixing region. In a closed vessel, all fluid particles stay inside the vessel for the whole duration of the mixing step. In an open-flow device such as in Fig. 16, fluid is constantly flowing out of the vicinity of the rods, due to the conservation of flow rate. Some of the escaping fluid has only spent a short while in the mixing region, because chaotic advection shuffles rapidly an incoming patch of dye onto the whole mixing region, including the part close to the outflow that goes with the main flow. This results in the dark dye filaments in Fig. 16 b), that have escaped shortly after their arrival in the mixing region and were not stretched enough to reach the Batchelor scale: mixing is therefore inefficient for such filaments with short residence times. On the other hand, fluid particles that stay for a longer time in the 
mixing region, as in Fig. $16 \mathrm{c}$ ) and d), are given enough time to reach the Batchelor scale and to diffuse with other concentration levels. Therefore, any measure of the efficiency of mixing of an open-flow protocol must focus on the fraction of fluid particles that escape the mixing region before they reach the Batchelor scale, like in Fig. 16 b); of course, this fraction should be as small as possible for a good quality of mixing.

\subsection{The geometry of dye mixing in open flows: chaotic saddle and strange eigenmode}

We have shown above that qualifying the mixing efficiency of an open-flow device imposes a distinction between short and long residence times, more precisely between weak and strong stretching, the limit being the stretching necessary for a patch of fluid to reach the Batchelor scale and diffuse. The organisation of the pattern of dye filaments and the transport in and out of the mixing region, including ill-mixed filaments, stems in fact from the geometry of the manifolds of the chaotic saddle, that were introduced in Sec. 3.

Fluid particles that are close to the stable manifold of the chaotic saddle approach the orbits of the chaotic saddle and shadow during some time their periodic trajectory. In a mixing experiment such as in Fig. 16, a few periods after the patch of dye entered the mixing region, only particles that were initially very close to the stable manifold of the chaotic saddle remain inside the mixing region. Therefore, the long-time dye filamentary pattern traces out the unstable manifold of the chaotic saddle, as in Fig. 16 (c) and (d). This mechanism was also sketched in Fig. 8 for a non-diffusive droplet. Nevertheless, the unstable manifold is a fractal one-dimensional set of zero measure, that is self-similar at all scales. Here, because of diffusion, grey filaments lie on the unstable manifold thickened to a width the Batchelor scale, which is the smallest length scale that can be observed in the pattern. Hence, the support of the dye is always this same set at long residence times. Moreover, we have shown in (Gouillart et al., 2009) that not only the support of the dye, but also the whole dye concentration field $C(\mathbf{x}, t)$, repeats perfectly over time, as in Fig. 16 (c) and (d). Only the global contrast of the pattern changes with time, because of the main flow that takes away a fraction of the fluid inside the mixing region at each stirring period.

$$
C(\mathbf{x}, t)=\langle C\rangle(t) \times \tilde{C}(\mathbf{x})
$$




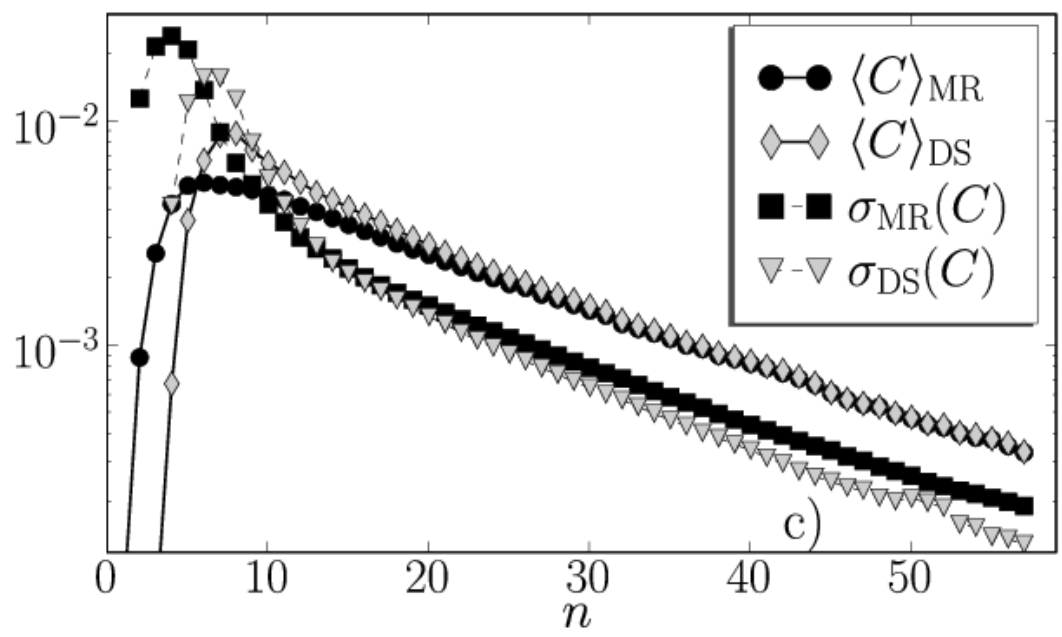

Figure 18: Typical evolution of the dye concentration mean and standard deviation for an open-flow protocol, inside the mixing region (MR, black symbols) and in the outflow (DS, grey symbols). Both moments of the concentration field have an exponential evolution with the same exponent after a short initial transient, because the dye concentration field takes a permanent form (Eq. (17)). 
Self-similar concentration patterns that repeat over time are observed in closed flows as well, where they have been termed strange eigenmodes (Pierrehumbert, 1994), because they correspond to the slowest eigenmode (except for the first trivial eigenmode, that is uniform) of the Perron-Frobenius advection-diffusion operator. Here, the permanent concentration pattern can be interpreted as well as an eigenmode of the advection-diffusion operator. Contrary to closed flows, the mean concentration inside the mixing region is not conserved because of the open flow. The time evolution of the open-flow eigenmode describes therefore the decay of the mean concentration of dye inside the mixing region:

$$
C(\mathbf{x}, t)=C_{\infty} \exp (-\kappa t) \tilde{C}(\mathbf{x})
$$

where $\kappa$ is the escape rate that appears in the Grassberger-Kantz relation (12). However, $\kappa$ characterises likewise the decay of all moments of the concentration field, as shown in Fig. 18 for the evolution of the mean $\langle C\rangle$ and the standard deviation $\sigma(C)$ of the concentration field. When measured inside the mixing region or in the outflow region, both quantities follow an exponential decay with the same decay rate, as imposed by Eq. (17). The constant ratio $\sigma(C) /\langle C\rangle$ is a measure of the normalised intensity of fluctuations inside the eigenmode pattern; this quantity can be used for defining a relevant measure of mixing efficiency, as we shall see later.

As the support of the strange eigenmode shadows the unstable manifold of the chaotic saddle, its concentration field also takes the fractal structure that was described in Section 3. In particular, holes in the support of the eigenmode (see Fig. 16 (c)) have a large variety of widths (the smallest possible width being the Batchelor scale). These holes do not contain any orbit of the chaotic saddle, neither portions of its unstable manifold. They correspond to the iterates of patches of fluid that entered the mixing region a short while ago: the larger the width, the more recent the entry time, as stretching by chaotic advection reduces the width of such patches. Fluid located in such holes, be it a patch of dyed or dye-less fluid, either ends up being compressed onto the unstable manifold fattened at a scale $w_{\mathcal{B}}$ if it stays in the mixing region for a sufficient time, or leaves the mixing region without having diffused with patches of fluid that entered the mixing region at a different time. In the outflow, the holes in the long-time permanent pattern therefore correspond to locations of ill-mixed fluid (no matter the composition of the inflow), while grey filaments correspond to well-mixed fluid. 
The fraction of well-mixed fluid may therefore be determined by measuring the fraction $\mathcal{A}$ of the outflow occupied by the support of the strange eigenmode, that is by the unstable manifold fattened by diffusion. In Fig. 6 (c) for example, the value of the well-mixed fraction for the blinking vortexsink flow is given by the fraction of the circle that leaves the mixing region at each half-period, that is occupied by the unstable manifold thickened to a finite diffusive width. In the channel rod-mixer of Fig. 16 (c), the well-mixed fraction is given in the same way by the fraction of the outflow pattern covered by grey filaments. In the inflow, fluid particles that will be well mixed are found within a distance $w_{\mathcal{B}}$ of the stable manifold. This is the uncertain region described in Section 4.3, with an uncertainty distance given by the diffusive length $w_{\mathcal{B}}$, and the unpredictability of trajectories that ensures good mixing for such fluid particles.

One should not measure the dye coverage inside the mixing region to compute the value of the well-mixed fraction, because in the mixing region some of the fluid inside the holes of the strange eigenmode pattern stays there for a time long enough to reach the Batchelor scale and be incorporated onto the strange eigenmode support. As a fraction of the mixing region is taken away to the outflow at each period (as the circle in Fig. 6 (c), or the upper part of the mixing region in Fig. 16 (c)), there is nevertheless a strong correlation between the long-time dye coverage inside the mixing region and the dye coverage in the outflow, that is, the well-mixed fraction. The strength of the correlation depends of the geometry of transport inside and out of the mixing region, that determines whether fluid in the largest holes leaves the mixing region before, after, or at the average residence time. In the same way, the fractal dimension of the unstable manifold that was introduced in Section 4 is correlated to the well-mixed fraction, as it measures the coverage of space by the unstable manifold; however, the large-scale organisation of the pattern and the sizes of its largest holes are not exactly characterised by the fractal dimension, which is defined at the small-scale limit.

An extreme counter-example of the correlation between the strength of mixing inside the mixing region and in the outflow is shown in Fig. 19. For this mixing experiment, the sense of rotation of the rods has been reversed compared with Fig. 16, while the direction of the main flow has been kept the same. Here, the motion of the rods accelerates fluid along the walls, so that an important fraction of the fluid never enters the mixing region and is not stretched by chaotic advection. We observe indeed on the long-time pattern (that is, the eigenmode pattern) in Fig. 19 that the unstable manifold covers 

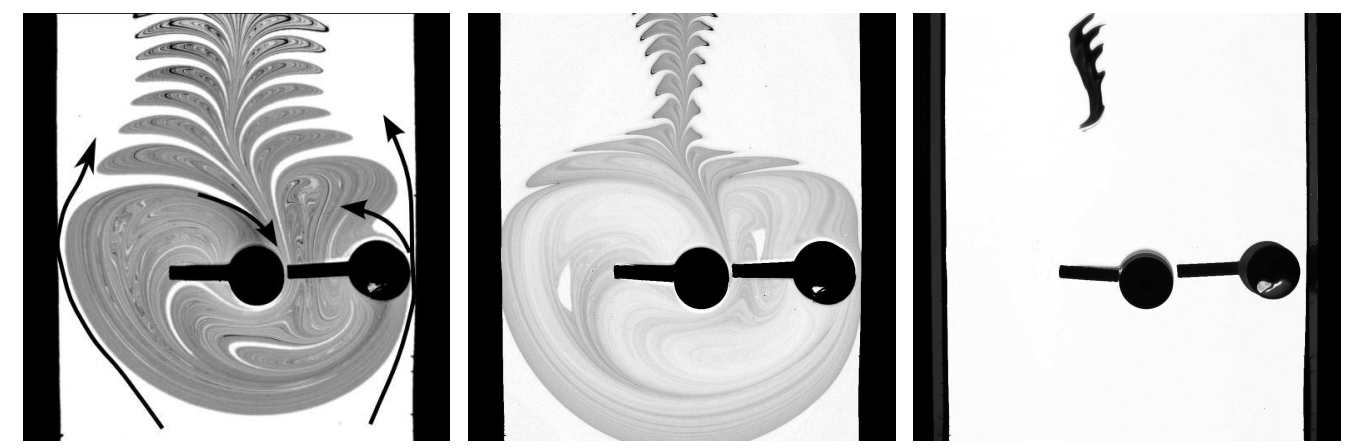

Figure 19: (a) Long-time dye pattern (strange eigenmode) for an open-flow protocol where rods accelerate fluid along the channel walls. Despite good mixing around the chaotic saddle, only a central strip of the outflow is well mixed, whereas fluid on the channel sides is not mixed at all. (b) Contrary to intuition, the quality of mixing decreases when the stirring frequency is increased, because the acceleration of fluid along the sides is enhanced. (c) Example of a patch of dye that crosses the channel without being mixed, because its initial position did not intersect the stable manifold of the chaotic saddle.

less than half the width of the channel - fluid flowing on the sides of the channel is hardly mixed at all. On the other hand, mixing inside the mixing region is very good, as the mixing region is covered very densely by dye filaments, with only a few thin holes. Measures of mixing should therefore be taken always in the outflow.

\subsection{The eigenmode index as a measure of mixing efficiency}

The knowledge gained on the link between the eigenmode and the different possible fates of fluid particles can now be used to derive a measure of mixing quality in open flows, that was dubbed eigenmode index in previous work (Gouillart et al., 2011). We have mentioned earlier that the fraction of the outflow $\mathcal{A}$ occupied by the support of the eigenmode is a direct measure of the fraction of fluid particles stretched to the Batchelor scale, and can therefore be used as a direct measure of mixing quality. In practice, this measure requires a hard threshold of the concentration value, and is therefore not a very robust one. The eigenmode index $\sigma_{\mathrm{SE}}$ defined below overcomes this problem, since it uses only the two first moments of the concentration 
distribution:

$$
\sigma_{\mathrm{SE}}=\frac{\sigma\left(C_{\mathrm{SE}}\right)}{\left\langle C_{\mathrm{SE}}\right\rangle}
$$

with $C_{\mathrm{SE}}(\mathbf{x})$ the concentration field of the eigenmode. $\sigma_{S E}$ and $\mathcal{A}$ are approximately related by

$$
\sigma_{\mathrm{SE}} \simeq \sqrt{\frac{1-\mathcal{A}}{\mathcal{A}}}
$$

$\sigma_{\mathrm{SE}}$ goes from zero for perfect mixing, to infinity for no mixing at all. As the definition of $\sigma_{\mathrm{SE}}$ uses only the eigenmode pattern, $\sigma_{\mathrm{SE}}$ does not depend on the position or size of an initial blob used for a mixing experiment (as long as the blob intersects the stable manifold of the chaotic saddle, so that the dye pattern traces out the strange eigenmode at long times - unlike the blob in Fig. 19 c), for example). For a fixed protocol of dye injection, it was shown in (Gouillart et al., 2011) that the eigenmode index is related to the intensity of segregation of chemical engineering (Danckwerts, 1952, 1953), a measure of mixing that compares the intensity of fluctuations in the outflow and in the inflow. The eigenmode index is nevertheless a more generic measure, as it does not depend on a specific inflow condition.

In theory, the eigenmode index can be measured from the concentration pattern in the outflow at any time after the Batchelor scale has been reached for all dye filaments that enter the outflow. In practice, it should be measured shortly after this diffusion time in order to keep a signal to noise ratio as high as possible, noise being inevitably present in any experiment. If possible, the contribution of noise (Gaussian white noise from the camera, bubbles or dust flowing with the fluid, etc.) to the standard deviation should be estimated, and removed from the measure of the standard deviation for the computation of $\sigma_{\mathrm{SE}}$. Also, $\sigma_{\mathrm{SE}}$ should be estimated on a region of the fluid that flows out of the mixing region during a single stirring period.

\subsection{Qualitative trends in mixing efficiency}

Let us now examine shortly the influence of a few physical parameters that control the efficiency of mixing.

First of all, Fig. 19 illustrates that the large-scale geometry of the flow is of paramount importance (here, only reverting the sense of rotation of the rods decreases greatly the efficiency of mixing). Bypasses around the mixing region should be avoided, as it is necessary that a particle passes close to an orbit of the chaotic saddle (if possible, at a distance less than the Batchelor scale) for being well mixed. 


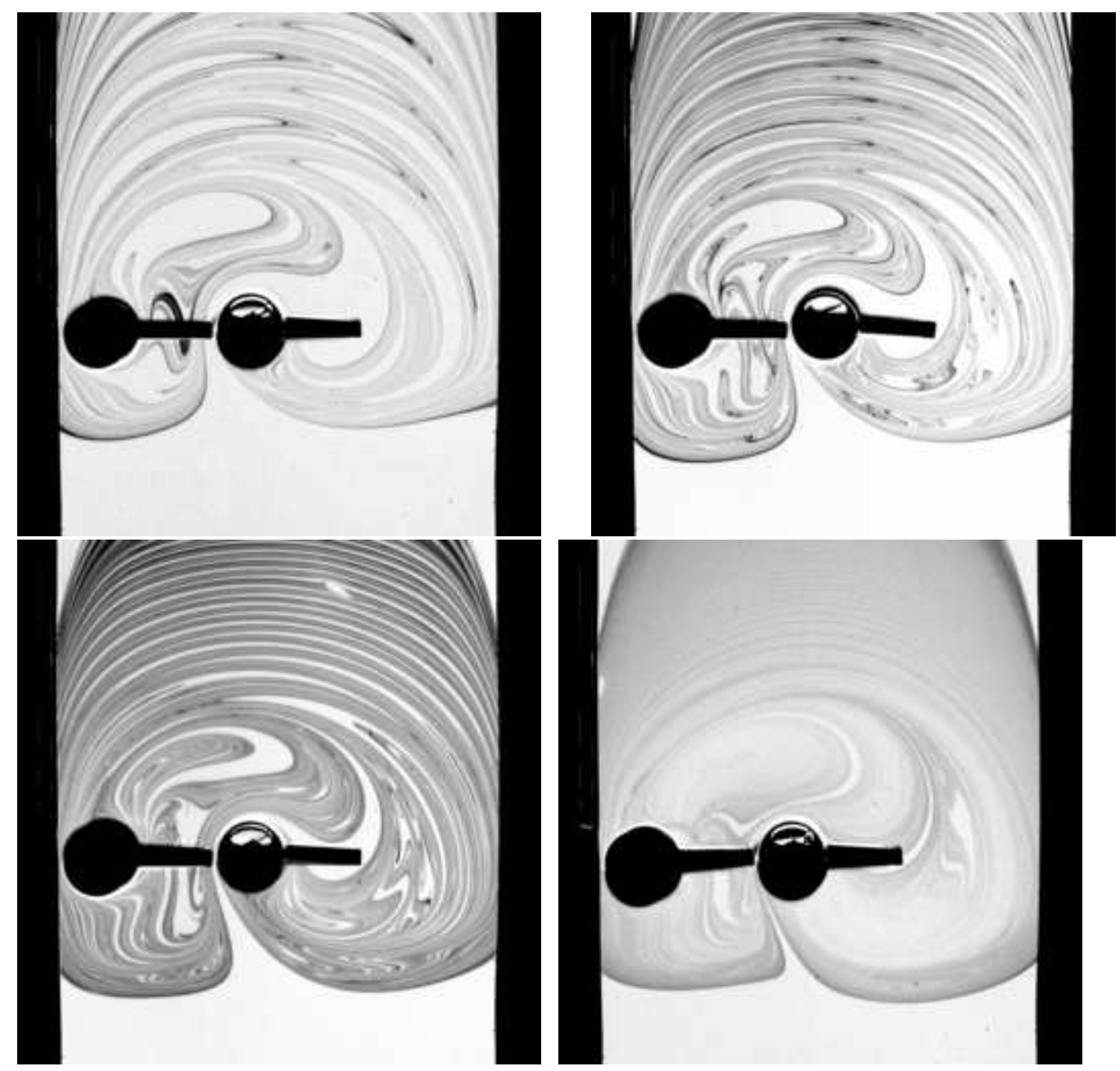

Figure 20: Mixing patterns at different stirring frequencies $(f=1,2,4,8$ $\mathrm{rpm})$. The dye-coverage of the outflow increases with the stirring frequency. 


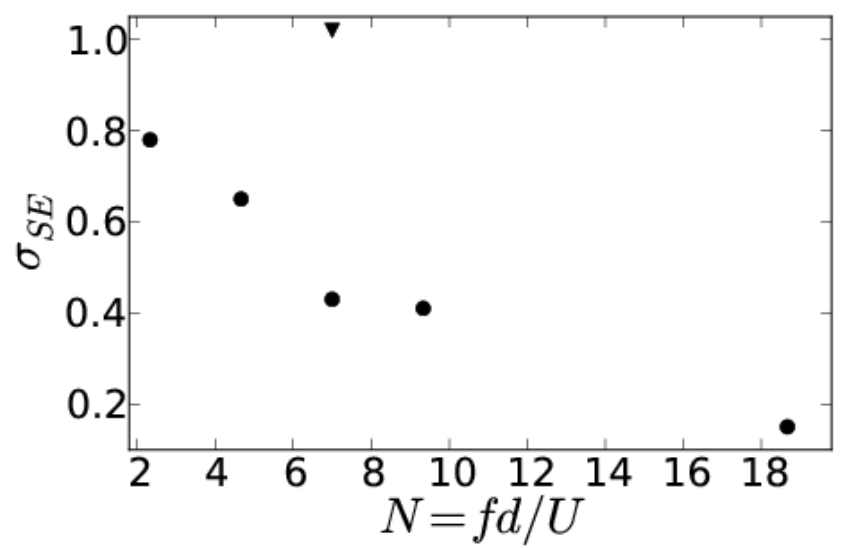

Figure 21: Eigenmode index $\sigma_{\mathrm{SE}}$ measured at different stirring frequencies $f$ for the rod-stirring protocol of Fig. 16 (circles). $\sigma_{\mathrm{SE}}$ is plotted against an approximate measure of the mean number of stirring periods $N$ spent by a fluid particle inside the mixing region. $N$ is computed from the extent of the trajectory of the rods $d$, and the mean velocity of the fluid $U$ in the main flow. The eigenmode index increases when the average residence time increases, since a greater fraction of the fluid reaches the Batchelor scale. Nevertheless, the geometry of the flow is also an important factor for the value of the eigenmode index, as shown by the high value (black triangle) of $\sigma_{\mathrm{SE}}$ when the direction of rotation of the rods is reversed (cf. Fig. 19)

Another important factor than can be easily tuned in an industrial setup is the average time of residency inside the mixing region. The mean number of stirring periods spent by a fluid particle inside the mixing region is controlled by the average velocity of the main flow $U$, the spatial extent of the mixing region $d$, and the stirring frequency $f$ :

$$
N=\frac{d f}{U}
$$

Increasing the average residence time inside the mixing region increases the fraction of fluid particles that are smeared by diffusion. We see indeed in Fig. 20 that the coverage of the outflow by the eigenmode (hence the efficiency of mixing) increases with the stirring frequency. This trend can be quantified by computing the eigenmode index, as shown in Fig. 21.

Slow stretching arising from non-hyperbolicity: elliptical islands and no-slip walls. Chaotic advection occurs in the vicinity of the orbits of the chaotic 
saddle, that cause exponential stretching of fluid particles. Nevertheless, for some protocols the dynamics of stretching are slower than exponential in a part of the mixing region, because of a non-hyperbolic chaotic saddle. A first case corresponds to KAM islands (see Section 5), that are segregated regions inside which fluid moves on regular trajectories and never escapes to the outflow, but for the weak action of diffusion only. KAM islands are observed in Fig. 22 (a-b) (one of them is pointed at by the red arrow in Fig. 22 a)), as small regions of dye-less fluid where dye never penetrates throughout the whole experiment. This is because fluid cannot cross the boundary between the chaotic region, and the islands. In Fig. 22 (a-b), we observe the stickiness of KAM islands that was illustrated in Section 5. The stickiness is shown by the dark dye filaments around the islands, that have been much less mixed with dye-less fluid than in the remainder of the chaotic region. At very long times (Fig. 22 (b)), the only visible fluctuations of the dye pattern are found around the sticky islands. Nevertheless, even if unmixed fluid stays around the islands, fluid particles are stretched to very fine filaments when they leave the vicinity of the islands, because the escape rate out of these regions is very weak. In Fig. 22 (b), dye filaments bear a high concentration level around the islands, yet no significant concentration fluctuation due to the escape of dye out of this region can be seen in the outflow. Elliptical islands therefore are only a minor issue in open flows.

A second case of non-hyperbolicity is shown in Fig. 22 c). When the rods pass close to the channel walls and the velocity of the main flow is small compared to the velocity of the rods, no fluid flows through the mixing region along the channel walls. The mixing region therefore extends to the walls of the channel, and the separation between the inflow and the mixing region is marked by two separation points on the channel walls (see Fig. 22 c)), and their unstable manifold. These separation points are parabolic points, which is a degenerate case, between hyperbolic orbits and elliptic KAM islands. Because of the fixed walls and the no-slip boundary condition, the stretching of fluid is very slow in the neighbourhood of the walls inside the mixing region. As a result, after a given time dye filaments have been much less mixed with dye-less fluid near the wall than in the bulk of the mixing region, as can be shown by the greater contrast of dye filaments near the wall (Fig. 22 c)). In closed flows, many authors (Chertkov and Lebedev, 2003; Gouillart et al., 2007, 2008; Salman and Haynes, 2007; Popovych et al., 2007; Boffetta et al., 2009) have shown that slow stretching at the wall has a dramatic effect on the rate of mixing. This is observed even in the bulk of the mixing region, 
because poorly-mixed fluid from the wall region periodically leaves the wall to be advected in the remainder of the chaotic region; in closed flows, it has therefore been argued that mixing can be more efficient if the chaotic region is insulated from the wall by a thin non-chaotic region (Gouillart et al., 2010). In open flows, however, the effect of walls is less dramatic, because even if poorly-mixed fluid is stored close to the walls, filaments that escape the vicinity of the wall do not flow directly to the outflow, but rather spend a few periods stretched inside the chaotic mixing region, exactly in the same way as other fluid particles that do not visit the vicinity of the walls. The main effect of walls is to reduce slightly the average value of stretching of the mixing region. Walls, therefore, may be considered as a slight inconvenience, but not as a primary cause of poor mixing in open flows, as they are in closed flows.

Note that regions with very long residence times are nevertheless an important drawback if almost stagnant patches of fluid evolve in an undesired way for long times (for example as a result of chemical evolution, or rheological evolution as for thixotropic fluids). A mixing region with more homogeneous stretching should be preferred for such cases.

Non-hyperbolic regions of anomalously slow (non-exponential) stretching also prevent the existence of a true concentration eigenmode, since fluid does not escape such regions at the same rate as for the remainder of the mixing region. Therefore, dye stays trapped there for longer times. For intermediate times, an almost invariant pattern is observed once dye filaments have reached the Batchelor scale in the bulk of the mixing region (Fig. 22 a) or c)) where stretching is exponential. At longer times however, the contrast of the dye pattern is localised on the regions of slow stretching (Fig. 22 b)). It has been observed indeed that concentration patterns do not converge on a permanent pattern in such cases, hence that the evolution of the concentration mean and standard deviation in the outflow do not obey exactly the same exponential law (Gouillart et al., 2009). For such protocols, the eigenmode index should be computed only at the intermediate times, where the main contribution to the standard deviation comes from the contrast between dye filaments of the bulk, and white holes of unmixed fluid.

In conclusion, good mixing, which is the stretching of fluid particles down to the Batchelor diffusion scale, is a difficult task in open flows because of the transient stay of fluid particles inside the mixing regions. While fluid particles with short residence times are often insufficiently mixed, fluid particles with long residence times are mixed much more than necessary. The 


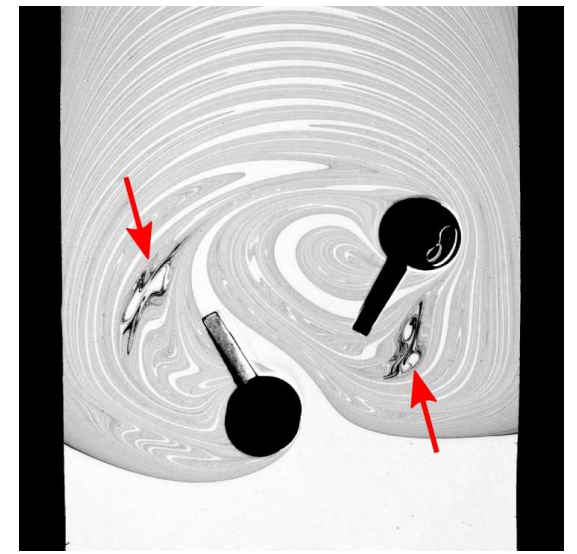

(a)
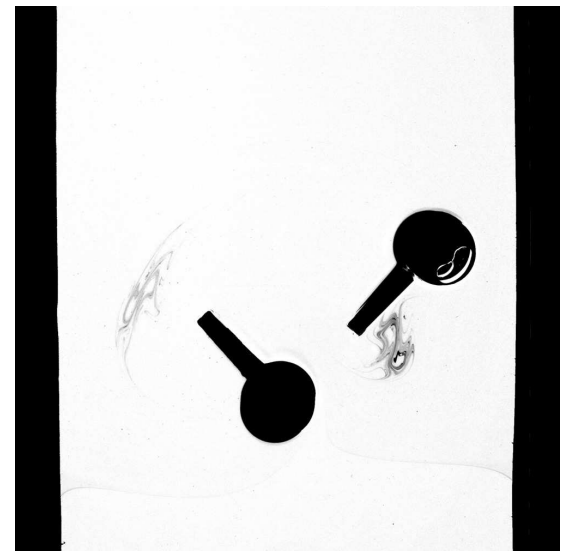

(b)

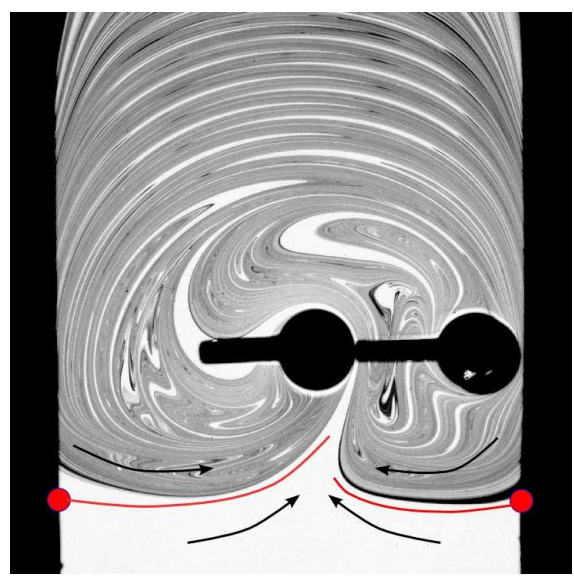

(c)

Figure 22: Mixing patterns with non-hyperbolic zones. (a) KAM islands (red arrows) are patches of fluid that stay forever inside the mixing region. Dye therefore never enters the islands. Islands have a "sticky" boundary where stretching is very low (hence the greater intensity of the dye) and dye is trapped for longer times than in the remainder of the mixing region. (b) At very long times after the entry of a blob of dye, dye is found only around the elliptical islands. (c) When the flow of the stirrers takes over the effect of the main channel flow near the walls of the channel, the mixing regions extends to the channel walls. Two parabolic separation points (red circles) and their unstable manifolds (red lines) define the limit between the inflow and the mixing region. Because of the no-slip condition, fluid particles close to the walls stay for long times inside the mixing region being hardly stretched. Therefore, unmixed fluid is stored near the walls and reinjected along the unstable manifold of the segaration points, as shown by the darker filaments. 
support of the eigenmode pattern, which is the unstable manifold of the chaotic saddle fattened at the diffusion scale, traces out the region where fluid is well-mixed, while its complementary corresponds to ill-mixed fluid. The normalised standard deviation of the eigenmode is therefore a relevant measure of mixing efficiency, called the eigenmode index.

\section{Chaotic advection in the ocean: plankton dynamics}

An important application of chaotic advection in open flows is the study of the interplay between mesoscale hydrodynamic motion and the distribution of marine organisms like phytoplankton and zooplankton. Since the seminal paper by Abraham (Abraham, 1998) this biological-physical interaction has been addressed in various studies (Mann and Lazier, 1991; Denman and Gargett, 1995; Peters and Marrasé, 2000; Károlyi et al., 2000; López et al., 2001a; Martin et al., 2002; Tél et al., 2005; Sandulescu et al., 2008). Several different aspects of plankton growth have been discussed, such as the emergence of sustainable plankton blooms (Hernández-García and López, 2004), localised plankton blooms in vortices (Sandulescu et al., 2007), the coexistence (Scheuring et al., 2003) and dominance (Bastine and Feudel, 2010) of species.

One of the major requirements for the growth of phytoplankton in the world's oceans is the availability of nutrients which is strongly dependent on the flow patterns in the oceans. Hence, the essential factors controlling the primary production, i.e. the growth of plankton, are horizontal and vertical transport of nutrients. Horizontal transport is influenced by the mesoscale hydrodynamic flow structures such as vortices and jet currents, while vertical transport is often related to coastal upwelling. The latter occurs usually when wind-driven currents, in combination with the Coriolis force, produces Ekman transport, by which surface waters are driven away from the coast and are replaced by nutrient-rich deep waters. Due to this upwelling the primary production in these areas is strongly enhanced, giving rise also to an increase of zooplankton and fish populations. Horizontal transport, mixing and stirring and its impact on plankton blooms has been investigated in several studies (Abraham, 1998; López et al., 2001b; Hernández-García et al., 2002, 2003; Martin, 2003). On the one hand, horizontal stirring by mesoscale structures like vortices and jets is responsible for the redistribution of plankton and nutrients and may therefore enhance primary production. On the other hand, horizontal transport can even cause the emergence of phytoplank- 
ton blooms (Károlyi et al., 2000). Furthermore, it influences competition and coexistence of different plankton species (Bracco et al., 2000).

The basic equations which govern the dynamics of plankton in the ocean contain three processes: (i) reactions modelling the biological growth of species, (ii) advection describing the advection of species by the flow, and (iii) diffusion accounting for the small scale turbulence not taken explicitly into account by the velocity field. The governing equations are reactiondiffusion-advection equations, which in case of a simple food chain model for the marine ecosystem consist of three equations for nutrients $N$, phytoplankton $P$ and zooplankton $Z$ :

$$
\begin{aligned}
& \frac{\partial N}{\partial t}+v \cdot \nabla N=F_{N}+D \Delta N \\
& \frac{\partial P}{\partial t}+v \cdot \nabla P=F_{P}+D \Delta P \\
& \frac{\partial Z}{\partial t}+v \cdot \nabla P=F_{Z}+D \Delta Z .
\end{aligned}
$$

To study the biological-physical interactions different models for the growth of the species $F_{N}, F_{P}, F_{Z}$ and different velocity fields $v$ have been used. The plankton dynamics include models which describe the plankton in terms of excitable systems (Truscott and Brindley, 1994; Neufeld et al., 2002) as well as of systems capable of exhibiting steady state and oscillatory behaviour (Edwards and Brindley, 1996). The velocity fields are either given by simple two-dimensional kinematic flows (Hernández-García and López, 2004) or in more realistic studies by ocean circulation models (Pasquero et al., 2004; Bracco et al., 2000). The diffusion term describes eddy diffusion, hence, all diffusion constants have the same value. Equations (21) are solved numerically by a semi-Lagrangian algorithm. That means, that the three processes, advection, reaction and diffusion, are performed sequentially. While the biological growth terms are treated as concentration changes on an Eulerian grid, the advection of fluid parcels is computed in a Lagrangian frame. The advantage of this procedure is that filamentary structures which are important features of chaotic advection of tracers, as pointed out in Sec. 4.2, can be resolved in a better way. For a detailed description of the integration scheme see (Sandulescu et al., 2008).

In the following we will discuss only one example for the biological and hydrodynamical models in order to demonstrate how the concept of chaotic advection can be used to explain the emergence of phytoplankton patterns 
as they are observed in satellite pictures. The filamentary and vortex-like structures seen in satellite observations can be largely explained as imprints of mesoscale hydrodynamical structures which enhance the emergence of plankton blooms. Particularly, we will highlight the role of the chaotic saddle, which as we have pointed out, is the most important feature of the mixing region in open flows (see Sec. 3).

The mixing regions in oceanic flows are characterised by mesoscale hydrodynamic structures. To study the impact of these mesoscale structures on plankton growth various kinematic models for the flow have been considered in literature. In general, only two-dimensional horizontal flow patterns have been studied so that the velocity field can be described by a stream function. This approach can be justified by the argument that vertical velocities in the ocean are often one order of magnitude smaller than the horizontal ones. Two paradigmatic models have been used to study the interplay between plankton dynamics and hydrodynamic flows: (i) the blinking vortex flow (Neufeld et al., 2002) depicted in Fig. 9, and (ii) the flow in the wake of an island (Jung et al., 1993), similar to the channel flow shown in Fig. 5. Both flows are periodically forced to ensure chaotic advection of passive tracers, as we have discussed in the previous sections. Each of them focuses on particular properties of a real flow. While (i) introduces a temporarily changing mixing region, (ii) mimics the dynamics of a von Kármán vortex street in the wake of an obstacle, which in geophysical flows can be considered as an island located in an ocean current.

Since the blinking vortex (i) flow is very idealised, only the flow in the wake of an island (ii) is discussed here in detail: The island is modelled as a cylinder located in a horizontal background flow. In the wake of this cylinder a von Kármán vortex street (cf. Fig.23) appears which is phenomenologically described by the following stream function $\Psi$ (Jung et al., 1993):

$$
\Psi(x, y, t)=f(x, y) g(x, y, t) .
$$

The first factor $f(x, y)$ ensures the correct boundary conditions at the cylinder, whereas the second factor $g(x, y, t)$ models the vortices in the wake, the background flow, and the Ekman flow:

$$
g(x, y, t)=w h_{1}(t) g_{1}(x, y, t)+w h_{2}(t) g_{2}(x, y, t)+u_{0} s(x, y) y+u_{E} \Theta(x-1) x .
$$

The vortices in the wake detach periodically from the island and their vorticity is of opposite sign. Their maximum vortex strengths denoted by $w$ 


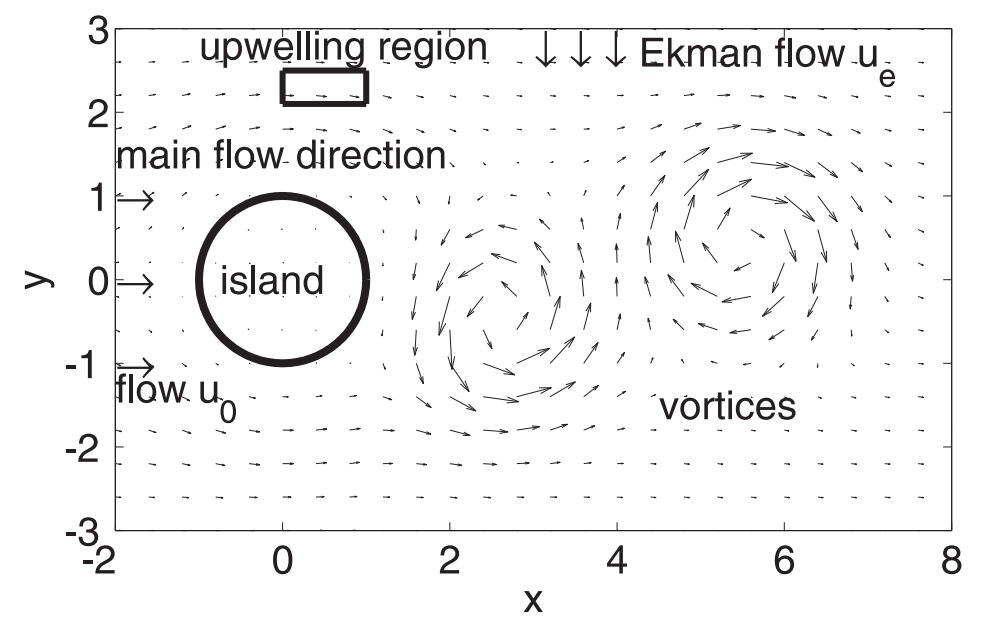

Figure 23: Sketch of the flow in the wake of an island. The upper border of the picture denotes the coastline.

are equal, and their shape is described by the functions $g_{i}(i=1,2)$ (see details in (Sandulescu et al., 2006)). Each vortex travels along the $x$ direction for a time $T_{c}$ before it disappears due to dissipation. The background flow moves in the same direction with a speed $u_{0}$. The factor $s(x, y)$ describes the shielding of the background flow by the cylinder in a phenomenological manner. The Ekman drift, which is intended to model the flow from the coast towards the ocean interior, is introduced by considering an additional velocity of constant strength $u_{E}$ in the $y$ direction which is perpendicular to the background flow and which is acting only at $x$ coordinates larger than 1 , i.e. just behind the island. This corresponds to a stream crossing the vortex street towards negative $y$ values beyond the cylinder.

The velocity components in $x$ and $y$ direction are then given by Eqs. (8), (22) and (23). The parametrisation of the model has been chosen in such a way that the hydrodynamic flow mimics the flow patterns around the Canary islands (Aristegui et al., 1997, 2004; Sandulescu et al., 2006).

From the large variety of marine ecosystem models we use a simple food chain to illustrate the interplay between hydrodynamic motion and biological 
growth. This model system is based on a three component model developed by Steele \& Henderson (Steele and Henderson, 1992) and later modified by Edwards \& Brindley (Edwards and Brindley, 1996) Pasquero et al. (Pasquero et al., 2004) and Oschlies \& Garcon (Oschlies and Garcon, 1999). The marine model ecosystem contains three different trophic levels, namely nutrients $N$, phytoplankton $P$ and zooplankton $Z$, whose concentrations evolve in time. The biological processes taken into account can be modelled as:

$$
\begin{aligned}
\frac{d N}{d t} & =\text { upwelling }- \text { uptake }+ \text { recycling } \\
\frac{d P}{d t} & =\text { uptake }- \text { grazing }- \text { mortality } \\
\frac{d Z}{d t} & =\text { grazing }- \text { mortality } .
\end{aligned}
$$

Expressed in mathematical terms this reads:

$$
\begin{aligned}
\frac{d N}{d t} & =F_{N}=\Phi_{N}-\beta \frac{N}{k_{N}+N} P+\mu_{N}\left[(1-\gamma) \frac{\alpha \eta P^{2}}{\alpha+\eta P^{2}} Z+\mu_{P} P+\mu Z^{2}\right] \\
\frac{d P}{d t} & =F_{P}=\beta \frac{N}{k_{N}+N} P-\frac{\alpha \eta P^{2}}{\alpha+\eta P^{2}} Z-\mu_{P} P \\
\frac{d Z}{d t} & =F_{Z}=\gamma \frac{\alpha \eta P^{2}}{\alpha+\eta P^{2}} Z-\mu Z^{2} .
\end{aligned}
$$

To provide some insight into the biological model we briefly discuss the meaning of the different terms in the model equations. For details we refer to (Oschlies and Garcon, 1999; Pasquero et al., 2004). The dynamics of the nutrients is determined by three processes, namely nutrient supply due to vertical mixing $\Phi_{N}$, consumption by phytoplankton $P$, and recycling by bacteria which are not explicitly taken into account as species in this model. Vertical mixing which brings nutrients from deeper nutrient-rich layers of the ocean into the mixed layer is parametrised in the biological model using the function $\Phi_{N}$, which depends on the abundance of nutrients $N$. This parametrisation is necessary because the two-dimensional hydrodynamical model takes only horizontal transport of nutrients into account. The uptake of nutrients by phytoplankton is described by a function which contains a saturation effect when nutrients are highly abundant. The recycling of nutrients from organic material (dead phytoplankton and zooplankton, exudates from zooplankton) is modelled by the term in brackets. 
The term describing the uptake of nutrients repeats as a growth term in the dynamical equation for phytoplankton $P$. Phytoplankton is diminished due to grazing by zooplankton and due to natural mortality.

Again the grazing term in the phytoplankton equation shows up as the growth term in the dynamical equation for the zooplankton $Z$, together with the conversion factor $\gamma$, which accounts for the fact that not all consumed phytoplankton are converted into biomass of zooplankton. The natural mortality of zooplankton is assumed to be quadratic because this term does not only model natural mortality but also the existence of higher predators which are not explicitly considered (Edwards and Bees, 2001). The parameters used in this model are taken from (Pasquero et al., 2004) and account for a situation in the open ocean.

Coupling the biological and the hydrodynamical model demonstrates how the concepts of chaotic advection can be used to contribute to the understanding of biogeochemical processes in the ocean. This simple conceptual model provides some insight into the mechanism of the interplay of mesoscale hydrodynamic structures, in this cases the vortices behind the island, and the biological growth of plankton species in the region of the vortex street. Depending on the concentrations of nutrients and plankton in the inflow into the area around the island one can distinguish two scenarios: (i) If the inflow of nutrients and plankton is rather high corresponding to a eutrophic ocean one observes an enhanced plankton growth in areas related to a large upwelling of nutrients (cf. Fig.24). Nutrients are entrained from the localised upwelling region where more nutrients are available due to higher vertical mixing rates and lead to enhanced plankton growth in this nutrient plume. This plankton bloom is essentially found in the exterior of the vortices. (ii) If the inflow of nutrients and plankton is rather low corresponding to an oligotrophic ocean the opposite behaviour is obtained. Instead of a plankton bloom surrounding the vortices one observes a localised plankton bloom within a vortex (cf. Fig.25). This rather different scenario which can be also observed in satellite pictures results from an intricate interplay between the time scales of the hydrodynamic and biological processes involved. Nutrients and plankton are entrained into the vortices in which the exchange of water with its surroundings is rather low. Hence, the plankton is confined to the vortex for a rather long time leading to an enhanced biological growth within the vortex. This way the vortex acts like an incubator for a plankton bloom. To unravel the mechanism how the nutrients and the plankton are transported into the vortex it is useful to study the details of the chaotic 


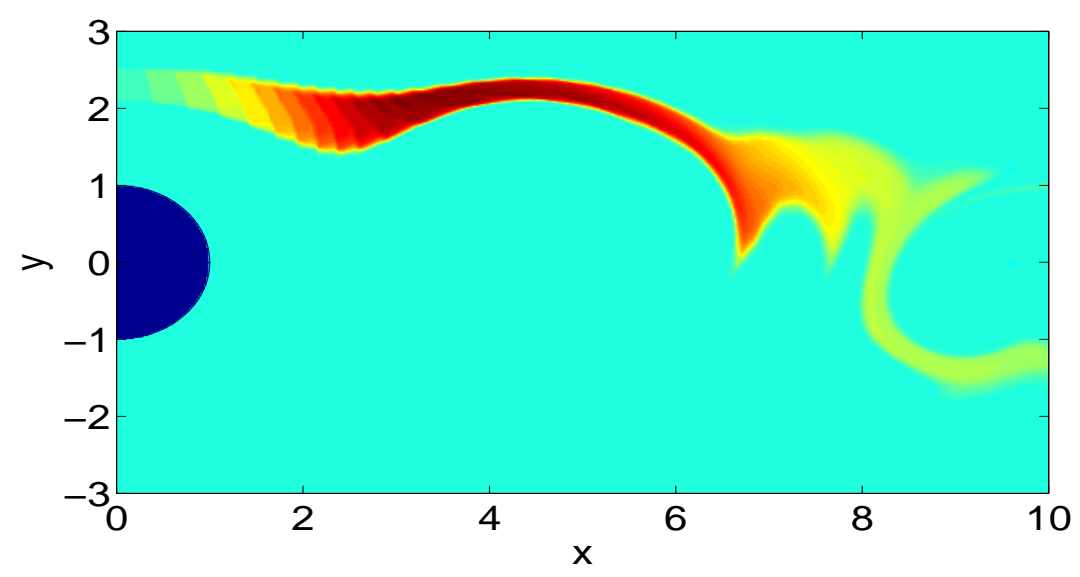

Figure 24: Snapshot of the phytoplankton concentration in the wake of an island with high inflow concentrations from the surrounding ocean. Concentrations from low to high are denoted by colours from blue via green to red.

advection of tracers in the flow Eqs. (8), (22) and (23).

Jung et al. (Jung et al., 1993) have shown that there exists a chaotic saddle in between the island and the vortex street. As pointed out in Sec. 3 , a chaotic saddle is an unstable invariant chaotic set possessing stable and unstable manifolds in a similar way as fixed point or periodic orbits of saddle type. Tracers in the neighbourhood of the stable manifolds move towards the chaotic saddle while tracers close to the chaotic saddle leave its neighbourhood along its unstable manifolds. Hence this chaotic saddle embedded in the flow can be considered as a "bridge" between the island and the vortex. Nutrients and plankton are transported along the stable manifolds from the vicinity of the island towards the chaotic saddle and from there into the vortex along its unstable manifolds. The chaotic saddle builds the backbone of transport between the immediate neighbourhood of the island and the interior of the vortex.

The transport of tracers along this path can be visualised using a concept from nonlinear dynamics, namely finite-size Lyapunov exponents (FSLE). This method has been proven to be very useful to investigate transport in open flows and illuminates the mechanism of the emergence of localised plankton blooms. The FSLEs allow for a characterisation of dispersion processes and for the detection of Lagrangian structures, such as barriers and 


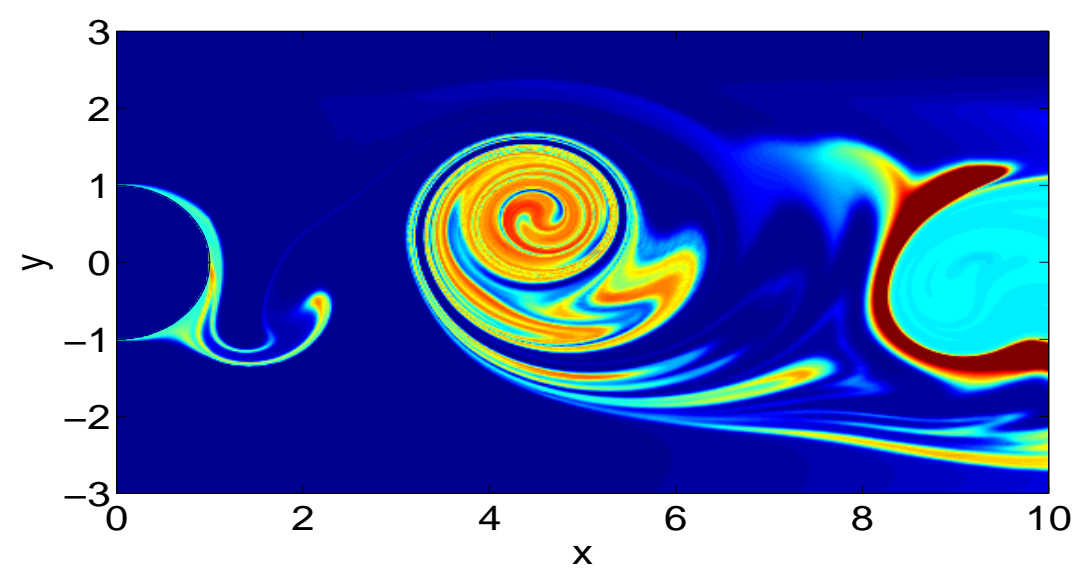

Figure 25: Snapshot of the phytoplankton concentration in the wake of an island with low inflow concentrations from the surrounding ocean. Concentrations from low to high are denoted by colours from blue via green to red.

vortices. Therefore the algorithm of their computation is briefly sketched in the following.

In dynamical systems theory the exponential divergence of initially nearby trajectories is in general measured by the computation of Lyapunov exponents. In order to adjust this concept to local processes in open flows, one computes finite size Lyapunov exponents (FSLE) which are based on the idea that one calculates the time which is necessary to reach a final prescribed distance $\delta_{f}$ starting from an initial distance $\delta_{0}$ (Artale et al., 1997; d'Ovidio et al., 2004). The FSLE are computed by starting two tracers in the flow at time $\mathrm{t}$ close to the point $x$ but at a small distance $\delta_{0}$, and let them evolve until their separation exceeds $\delta_{f}$. From the elapsed time, $\tau_{ \pm}$, the FSLE is calculated as

$$
\lambda_{ \pm}\left(x, t, \delta_{0}, \delta_{f}\right)=\frac{1}{\tau_{ \pm}} \log \frac{\delta_{f}}{\delta_{0}}
$$

The positive subscripts indicate that the tracers are advected forward in time, while for the negative subscript they are advected backward in time. $\lambda_{ \pm}$is a scalar measure for the stretching/contracting rate in the flow given by the inverse of the separation time $\tau_{ \pm}$. Maxima in the spatial distribution of $\lambda_{+}$, the positive or expanding FSLE, approximate the underlying stable manifold of the chaotic flow (Joseph and Legras, 2002; d'Ovidio et al., 2004), the direction along which tracers approach the saddle. The contracting FSLE, 


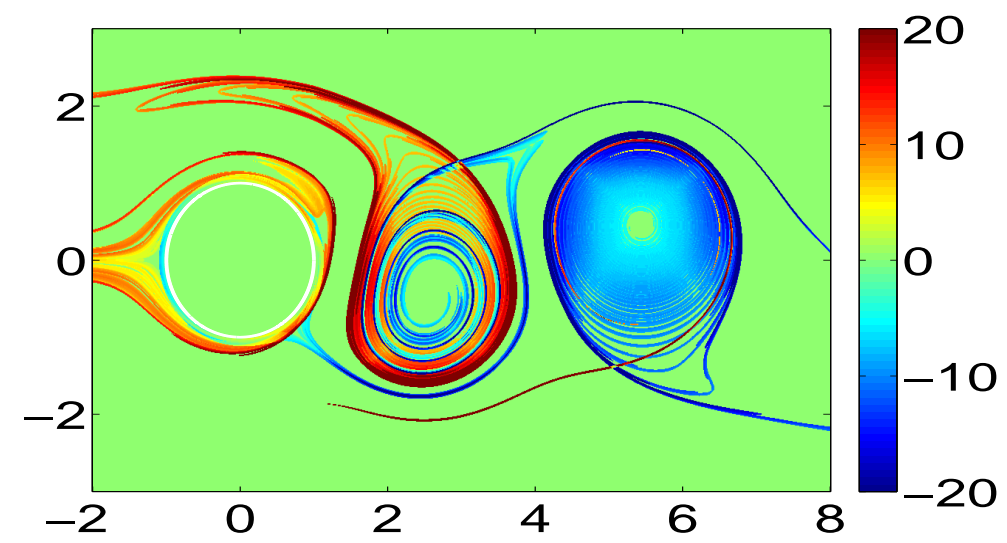

Figure 26: Snapshot of the stable (red) and unstable (blue) manifolds in the wake of the island.

$\lambda_{-}$, detects the underlying unstable manifold in the flow, the direction along which tracers leave the saddle. The stable and unstable manifolds are intertwined around the vortex cores and at the island (Fig. 26). Their complex structure allows for transport of tracers across the vortex street as well as of nutrients and plankton into the interior of the vortex. The results of this approach reveal one possible mechanism for the emergence of localised plankton blooms which can be observed by satellite in many different areas around the globe.

\section{Summary}

The transient nature of chaotic advection in open flows results in partial mixing organised around persistent (non-space-filling) fractal patterns in the mixing region. These fractal structures are caused by the presence of the chaotic saddle in the mixing region, and the invariant manifolds associated with the chaotic saddle govern the dynamics of the system. The chaotic saddle is felt not only in pure advection, but also in the case of transported scalars subject to molecular diffusion; the strange eigenmode which appears in the asymptotic dynamics of the scalar concentration shadows the unstable manifold for low diffusion. The chaotic saddle and its unstable manifold are also the key to understanding the dynamics of chemical and biological processes in fluids, as most of the reactions take place in the vicinity of the unstable manifold. In conclusion, the concept of the chaotic saddle is 
the unifying principle behind the theoretical and experimental approaches to phenomena related to chaotic advection.

\section{Bibliography}

Abraham, E., 1998. The generation of plankton patchiness by turbulent stirring. Nature 391, 577.

Abraham, E.R., Law, C.S., Boyd, P.W., Lavender, S.J., Maldonado, M.T., Bowie, A.R., 2000. Importance of stirring in the development of an ironfertilized phytoplankton bloom. Nature 407, 727.

Aref, H., 1984. Stirring by chaotic advection. J. Fluid Mech. 143, 1.

Aref, H., Jones, S.W., Mofina, S., Zawadzki, I., 1989. Vortices, kinematics and chaos. Physica D 37, 423.

Aristegui, J., Barton, E.D., Tett, P., Montero, M.F., García-Munoz, M., Basterretxea, G., Cussatlegras, A.S., Ojeda, A., de Armas, D., 2004. Variability in plankton community structure, metabolism, and vertical carbon fluxes along an upwelling filament. Prog. Oceanogr. 62, 95.

Aristegui, J., Tett, P., Hernández-Guerra, A., Basterretxea, G., Montero, M.F., Wild, K., Sangrá, P., Hernández-Leon, S., Canton, M., GarcíaBraun, J.A., Pacheco, M., Barton, E., 1997. The influence of islandgenerated eddies on chlorophyll distribution: a study of mesoscale variation around gran canaria. Deep-Sea Res. I 44, 71.

Artale, V., Boffetta, G., Celani, M., Cencini, M., Vulpiani, A., 1997. Dispersion of passive tracers in closed basins: Beyond the diffusion coefficient. Phys. Fluids 9, 3162.

Aubin, J., Fletcher, D., Bertrand, J., Xuereb, C., 2003. Characterization of the mixing quality in micromixers. Chem. Engin. Tech. 26, 1262.

Bastine, D., Feudel, U., 2010. Inhomogeneous dominance patterns of competing phytoplankton groups in the wake of an island. Nonlin. Proc. Geophys. 17,715 .

Batchelor, G.K., 1959. Small-scale variation of convected quantities like temperature in turbulent fluid: Part 1. General discussion and the case of small conductivity. J. Fluid Mech. 5, 113. 
Batchelor, G.K., 1967. An introduction to fluid dynamics. Cambridge University Press, Cambridge.

Benczik, I.J., Toroczkai, Z., Tél, T., 2002. Selective sensitivity of open chaotic flows on inertial tracer advection: catching particles with a stick. Phys. Rev. Lett. 89, 164501.

Biemond, J.J.B., de Moura, A.P.S., Károlyi, G., Grebogi, C., Nijmeijer, H., 2008. Onset of chaotic advection in open flows. Phys. Rev. E 78, 016317.

Boffetta, G., De Lillo, F., Mazzino, A., 2009. Peripheral mixing of passive scalar at small Reynolds number. J. Fluid Mech. 624, 151.

Boyd, P.W., Watson, A.J., Law, C.S., Abraham, E.R., Trull, T., Murdoch, R., Bakker, D.C., Bowie, A.R., Buesseler, K.O., Chang, H., Charette, M., Croot, P., Downing, K., Frew, R., Gall, M., Hadfield, M., Hall, J., Harvey, M., Jameson, G., LaRoche, J., Liddicoat, M., Ling, R., Maldonado, M.T., McKay, R.M., Nodder, S., Pickmere, S., Pridmore, R., Rintoul, S., Safi, K., Sutton, P., Strzepek, R., Tanneberger, K., Turner, S., Waite, A., Zeldis, J., 2000. A mesoscale phytoplankton bloom in the polar southern ocean stimulated by iron fertilization. Nature 407, 695 .

Bracco, A., Provenzale, A., Scheuring, I., 2000. Mesoscale vortices and the paradox of the plankton. Proc. Roy. Soc. Lond. B 267, 1795.

Bryant, J., 1977. The characterization of mixing in fermenters. Adv. Biochem. Eng 5, 101.

Cartwright, J.H.E., Feingold, M., Piro, O., 1996. Chaotic advection in threedimensional unsteady incompressible laminar flow. J. Fluid Mech. 316, 259.

Cartwright, J.H.E., Feudel, U., Karolyi, G., Moura, A., Piro, O., Tel, T., 2010. Nonlinear dynamics and chaos: advances and perspectives. SpringerVerlag. chapter Dynamics of finite-size particles in chaotic fluid flows. p. 51.

Chertkov, M., Lebedev, V., 2003. Boundary effects on chaotic advectiondiffusion chemical reactions. Phys. Rev. Lett. 90, 134501.

Danckwerts, P.V., 1952. The definition and measurement of some characteristics of mixtures. Appl. Sci. Res. A3, 279. 
Danckwerts, P.V., 1953. Continuous flow systems-distribution of residence times. Chem. Eng. Sci. 2, 1.

Denman, K., Gargett, A., 1995. Biological-physical interactions in the upper ocean: the role of vertical and small scale transport processes. Annu. Rev. Fluid Mech. 27, 225.

d'Ovidio, F., Fernández, V., Hernández-Garcia, E., López, C., 2004. Mixing structures in the mediterranean sea from finitesize lyapunov exponents. Geophys. Res. Lett. 31, L17203.

Edwards, A.M., Bees, M., 2001. Generic dynamics of a simple plankton population model with a non-integer exponent of closure. Chaos Solitons Fractals 12, 289.

Edwards, A.M., Brindley, J., 1996. Oscillatory behavior in a three component plankton population model. Dyn. Stab. Sys. 11, 347.

Ehrfeld, W., Golbig, K., Hessel, V., Lowe, H., Richter, T., 1999. Characterization of mixing in micromixers by a test reaction: single mixing units and mixer arrays. Ind. Eng. Chem. Res 38, 1075.

Falconer, K., 2003. Fractal Geometry: Mathematical Foundations and Applications. Wiley, New York.

Gouillart, E., Dauchot, O., Dubrulle, B., Roux, S., Thiffeault, J.L., 2008. Slow decay of concentration variance due to no-slip walls in chaotic mixing. Phys. Rev. E 78, 026211.

Gouillart, E., Dauchot, O., Thiffeault, J., 2011. Measures of mixing quality in open flows with chaotic advection. Phys. Fluids 23, 3604.

Gouillart, E., Dauchot, O., Thiffeault, J.L., Roux, S., 2009. Open-flow mixing: Experimental evidence for strange eigenmodes. Phys. Fluids 21, 023603.

Gouillart, E., Kuncio, N., Dauchot, O., Dubrulle, B., Roux, S., Thiffeault, J.L., 2007. Walls inhibit chaotic mixing. Phys. Rev. Lett. 99, 114501.

Gouillart, E., Thiffeault, J., Dauchot, O., 2010. Rotation Shields Chaotic Mixing Regions from No-Slip Walls. Phys. Rev. Lett. 104, 204502. 
Grassberger, P., Kantz, H., 1985. Repellers, semi-attractors, and long-lived chaotic transients. Physica D 17, 75.

Halsey, Jensen, Kadanoff, Procaccia, Shraiman, 1986. Fractal measures and their singularities: The characterization of strange sets. Phys. Rev. A 33, 1141.

Hernández-García, E., López, C., 2004. Sustained plankton blooms under open chaotic flows. Ecol. Complex. 1, 253.

Hernández-García, E., López, C., Neufeld, Z., 2002. Small-scale structure of nonlinearly interacting species advected by chaotic flows. Chaos 12, 470 .

Hernández-García, E., López, C., Neufeld, Z., 2003. Spatial patterns in chemically and biologically reacting flows, in: Bofetta, G., Lacorata, G., Visconti, G., Vulpiani, A. (Eds.), Chaos in geophysical flows. OTTO Editore, Torino, pp. 35-61.

Joseph, B., Legras, B., 2002. Relation between kinematic boundaries, stirring, and barriers for the antartic polar vortex. J. Atmos. Sci. 59, 1198.

Jung, C., Tél, T., Ziemniak, E., 1993. Application of scattering chaos to particle transport in a hydrodynamical flow. Chaos 3, 555 .

Károlyi, G., Péntek, A., Scheuring, I., Tél, T., Toroczkai, Z., 2000. Chaotic flow: the physics of species coexistence. Proc. Natl. Acad. Sci. USA 97, 13661.

Károlyi, G., Tél, T., 1997. Chaotic tracer scattering and fractal basin boundaries in a blinking vortex-sink system. Phys. Rep. 290, 125.

Koh, T.Y., Legras, B., 2002. Hyperbolic lines and the stratospheric polar vortex. Chaos 12, 382 .

Kukukova, A., Aubin, J., Kresta, S., 2009. A new definition of mixing and segregation: Three dimensions of a key process variable. Chem. Eng. Res. Des. 87,633 .

Károlyi, G., Tél, T., de Moura, A.P.S., Grebogi, C., 2004. Reactive particles in random flows. Phys. Rev. Lett. 92, 174101.

Lai, Y.C., Tél, T., 2011. Transient chaos. Springer-Verlag, New York. 
Landau, L., Lifshits, E., 2000. Fluid Mechanics. Elsevier, Oxford.

Lau, Y.T., Finn, J.M., Ott, E., 1991. Fractal dimension in nonhyperbolic chaotic scattering. Phys. Rev. Lett. 66, 978.

Lee, Y., Deval, J., Tabeling, P., Ho, C., 2001. Chaotic mixing in electrokinetically and pressure driven micro flows, in: 14th IEEE International conference on micro-electro-mechanical systems, p. 483.

López, C., Hernández-García, E., Piro, O., Vulpiani, A., Zambianchi, E., 2001a. Population dynamics advected by chaotic flows: A discrete-time map approach. Chaos 11, 397.

López, C., Neufeld, Z., Hernández-García, E., Haynes, P., 2001b. Chaotic advection of reacting substances: Plankton dynamics on a meandering jet. Phys. Chem. Earth, B 26, 313.

MacKay, R.S., Meiss, J.D. (Eds.), 1987. Hamiltonian dynamical systems. Institue of Physics Publishing, London.

Mann, K., Lazier, J., 1991. Dynamics of marine ecosystems, Biologicalphysical interactions in the oceans. Blackwell Scientific Publications, Boston.

Martin, A., 2003. Phytoplankton patchiness: the role of lateral stirring and mixing. Prog. Oceanogr. 57, 125.

Martin, A., Richards, K., Bracco, A., Provenzale, A., 2002. Patchy productivity in the open ocean. Global Biogeochem. Cy. 16, 1025.

Meiss, Ott, 1985. Markov-tree model of intrinsic transport in hamiltonian systems. Phys. Rev. Lett. 55, 2741.

Motter, A.E., Lai, Y.C., Grebogi, C., 2003. Reactive dynamics of inertial particles in nonhyperbolic chaotic flows. Phys. Rev. E 68, 056307.

Motter, A.E., de Moura, A.P.S., Grebogi, C., Kantz, H., 2005. Effective dynamics in hamiltonian systems with mixed phase space. Phys. Rev. E 71, 036215 .

de Moura, A.P.S., Grebogi, C., 2004a. Chemical and biological activity in three-dimensional flows. Phys. Rev. E 70, 026218. 
de Moura, A.P.S., Grebogi, C., 2004b. Reactions in flows with nonhyperbolic dynamics. Phys. Rev. E 70, 036216.

Neufeld, Z., López, C., Hernández-García, E., Piro, O., 2002. Excitable media in open and closed chaotic flows. Phys. Rev. E 66, 066208.

Okkels, F., Tabeling, P., 2004. Spatiotemporal resonances in mixing of open viscous fluids. Phys. Rev. Lett. 92, 038301.

Oschlies, A., Garcon, V., 1999. An eddy-permitting coupled physicalbiological model of the north-atlantic, sensitivity to advection numerics and mixed layer physics. Global Biogeochem. Cy. 13, 135.

Ott, E., 1993. Chaos in dynamical systems. Cambridge University Press, Cambdridge.

Ottino, J.M., 1989. The Kinematics of Mixing: Stretching, Chaos and Transport. Cambridge University Press, Cambridge.

Pasquero, C., Bracco, A., Provenzale, A., 2004. Coherent vortices, lagrangian particles and the marine ecosystem, in: Uijttewaal, W., Jirka, G. (Eds.), Shallow Flows. Balkema Publishers, Leiden, p. 399.

Paul, E.L., Atiemo-Obeng, V., Kresta, S.M. (Eds.), 2003. Handbook of Industrial Mixing: Science and Practice. Wiley.

Pedlosky, J., 1979. Geophysical Fluid Dynamics. Springer, Berlin.

Peters, F., Marrasé, C., 2000. Effects of turbulence on plankton: an overview of experimental evidence and some theoretical considerations. Mar. Ecol. Prog. Ser. 205, 291.

Pierrehumbert, R.T., 1994. Tracer microstructure in the large-eddy dominated regime. Chaos Solitons Fractals 4, 1091.

Popovych, O.V., Pikovsky, A., Eckhardt, B., 2007. Abnormal mixing of passive scalars in chaotic flows. Phys. Rev. E 75, 036308.

Rodrigues, C.S., de Moura, A.P.S., Grebogi, C., 2010. Random fluctuation leads to forbidden escape of particles. Phys. Rev. E 82, 026211. 
Salman, H., Haynes, P.H., 2007. A numerical study of passive scalar evolution in peripheral regions. Phys. Fluids 19, 067101.

Sandulescu, M., Hernández-García, E., López, C., Feudel, U., 2006. Kinematic studies of transport across an island wake, with application to the canary islands. Tellus A 58, 605.

Sandulescu, M., Hernández-García, E., López, C., Feudel, U., 2007. Kinematic studies of transport across an island wake, with application to the canary islands. Nonlin. Processes Geophys. 14, 443.

Sandulescu, M., López, C., Hernández-García, E., Feudel, U., 2008. Biological activity in the wake of an island close to a coastal upwelling. Ecol. Complex. 5, 228.

Schelin, A.B., Károlyi, G., de Moura, A.P.S., Booth, N.A., Grebogi, C., 2009. Chaotic advection in blood flow. Phys. Rev. E 80, 016213.

Schelin, A.B., Károlyi, G., de Moura, A.P.S., Booth, N.A., Grebogi, C., 2010. Fractal structures in stenoses and aneurysms in blood vessels. Philos. Transact. Roy. Soc. A 368, 5605.

Scheuring, I., Károlyi, G., Toroczkai, Z., Tél, T., Péntek, A., 2003. Competing populations in flows with chaotic mixing. Theor. Pop. Biol. 63, 77.

Sommerer, J.C.e.a., 1996. Experimental evidence for chaotic scattering in a fluid wake. Phys. Rev. Lett. 77, 5055.

Steele, J., Henderson, E., 1992. The role of predation in plankton models. J. Plankton Res. 14, 157.

Stroock, A.D., Dertinger, S.K.W., Adjari, A., Mezić, I., Stone, H.A., Whitesides, G.M., 2002. Chaotic mixer for microchannels. Science 295, 647.

Taylor, G., 1953. Dispersion of soluble matter in solvent flowing slowly through a tube. Proc. Roy. Soc. Lond. A 219, 186.

Tél, T., de Moura, A., Grebogi, C., Károlyi, G., 2005. Chemical and biological activity in open flows: a dynamical systems approach. Phys. Rep. 413, 91. 
Truscott, J.E., Brindley, J., 1994. Ocean plankton populations as excitable media. Bull. Math. Biol. 56, 981.

Tuval, I., Schneider, J., Piro, O., Tel, T., 2004. Opening up fractal structures of three-dimensional flows via leaking. Europhys. Lett. 65, 633.

Vilela, R.D., de Moura, A.P.S., Grebogi, C., 2006. Finite-size effects on open chaotic advection. Phys. Rev. E 73, 026302.

Vilela, R.D., Tél, T., de Moura, A.P.S., Grebogi, C., 2007. Signatures of fractal clustering of aerosols advected under gravity. Phys. Rev. E 75, 065203.

Villermaux, E., Duplat, J., 2003. Mixing as an aggregation process. Phys. Rev. Lett. 91, 184501. 